\title{
The pH Dependence of Niclosamide Solubility, Dissolution, and Morphology: Motivation for Potentially Universal Mucin-Penetrating Nasal and Throat Sprays for COVID I9, its Variants and other Viral Infections
}

\author{
David Needham ${ }^{1,2}$ (D)
}

Received: 13 August 2021 / Accepted: 14 September 2021 / Published online: 28 December 2021

(C) Springer Science+Business Media, LLC, part of Springer Nature 2021

\begin{abstract}
Motivation With the coronavirus pandemic still raging, prophylactic-nasal and early-treatment throatsprays could help prevent infection and reduce viral load. Niclosamide has the potential to treat a broadrange of viral infections if local bioavailability is optimized as mucin-penetrating solutions that can reach the underlying epithelial cells.
\end{abstract}

Experimental pH-dependence of supernatant concentrations and dissolution rates of niclosamide were measured in buffered solutions by UV/Vis-spectroscopy for niclosamide from different suppliers (AK Sci and Sigma), as precipitated material, and as cosolvates. Data was compared to predictions from HendersonHasselbalch and precipitation-pH models. Opticalmicroscopy was used to observe the morphologies of original, converted and precipitated niclosamide.

Results Niclosamide from the two suppliers had different polymorphs resulting in different dissolution behavior. Supernatant concentrations of the "AKScipolymorph" increased with increasing $\mathrm{pH}$, from $2.53 \mu \mathrm{M}$ at $\mathrm{pH} 3.66$ to $300 \mu \mathrm{M}$ at $\mathrm{pH} 9.2$, reaching $703 \mu \mathrm{M}$ at $\mathrm{pH}$

David Needham

d.needham@duke.edu

1 Department of Mechanical Engineering and Material Science, Duke University, Durham, North Carolina 27708, USA

2 Professor of Translational Therapeutics, School of Pharmacy, University of Nottingham, Nottingham NG7 2RD, UK
9.63. However, the "Sigma-polymorph" equilibrated to much lower final supernatant concentrations, reflective of more stable polymorphs at each pH. Similarly, when precipitated from supersaturated solution, or as cosolvates, niclosamide also equilibrated to lower final supernatant concentrations. Polymorph equilibration though was avoided by using a solvent-exchange technique to make the solutions.

Conclusions Given niclosamide's activity as a host cell modulator, optimized niclosamide solutions could represent universal prophylactic nasal and early treatment throat sprays against COVID19, its more contagious variants, and other respiratory viral infections. They are the simplest and potentially most effective formulations from both an efficacy standpoint as well as manufacturing and distribution, (no cold chain). They now just need testing.

KEY WORDS COVID19 - host cell modulation · niclosamide-solubility · pH-dependent-dissolution • universal nasal-throat-spray $\cdot$ variants $\cdot$ viral infections

\section{INTRODUCTION}

\section{Study Overview}

This article lays out the case for new universal prophylactic nasal and early treatment throat sprays for COVID19, its more contagious variants (1), and other respiratory viral infections. The sprays are based on aqueous solutions of the anti-helminthic drug niclosamide, a drug with relatively low water solubility at pH 7 (and hence prohibitively low bioavailability). A 
preformulation drug characterization of niclosamide (pKa, water-solubility, and $\log \mathrm{P}$ ) was carried out along with an experimental evaluation of the amount of niclosamide that could be dissolved in aqueous solution as a function of $\mathrm{pH}$. Results revealed that the concentration of niclosamide in aqueous solution can be increased by simply increasing solution $\mathrm{pH}$ by only one to two logs, into the $\mathrm{pH} 8-9$ range. However, studies also showed that, in the presence of solid phase niclosamide, the amount of niclosamide in solution is determined by the solid state nature of the material (polymorph) that the aqueous solution is in equilibrium with or is moving towards (2). Not every source of niclosamide (AKSci (CA) and Sigma (MO) is equal in polymorphic nature, intrinsic solubility, and therefore in vivo therapeutic performance. Such solid phase material includes precipitated or particulate niclosamide as recently used as micronized (3) or stabilized microparticles. Our lead niclosamide formulation is therefore a simple buffered mucin-penetrating solution with optimized bioavailability because it does not contain any microparticles of niclosamide. It represents a low dose prophylactic nasal spray that, according to recent literature on niclosamide and viruses (4-19), could stop virus replication at its point of entry in the nose, and a higher concentration throat spray, that could reduce viral load as it progresses down the back of the throat.

\section{Niclosamide Inhibits Three of the Six Stages of Viral Infection}

Why niclosamide? Niclosamide, marketed as Yomesan by Bayer (20) and other generics, has been routinely given to humans as oral tablets in a 2-g dose to cure cestode parasites (tape worms) for the past 60 years (21). Over the past 10 years, it has emerged from multiple drug screens as a very interesting compound; not just as a pesticide but also with potential for cancer and many other diseases and conditions (22), and now a broad range of viral infections (4-19). The reason for this broad-range activity is that, because viruses are obligate intracellular pathogens, they cannot replicate without utilizing the machinery and metabolism of a host cell. As discussed by Goulding (23), there are six basic stages that are essential for viral replication at the cellular level: 1. Attachment, 2. Penetration, 3. Uncoating, 4. Replication, 5. Assembly, and 6. Release of the mature virions. As reported in the literature for various viruses, (4-19), Niclosamide can inhibit three of these, namely, 3. Uncoating, that prevents viral RNA release from the endosome; 4 . Replication, where it reduces the amount of ATP available from host cell mitochondria and so inhibits the viral transcription and translation events that are ATP dependent; and 5. Assembly in the Golgi that then results in the secretion of non-competent virions.

While many current anti-virals either attempt to disrupt the synthesis and assembly of viral proteins or target host proteins and mechanisms required by the viral replication cycle (24), reformulated niclosamide offers a different, and potentially very effective, way to prevent and combat early viral infection. As discussed in more detail in a white paper in preparation (25), it enters cell membranes as a lipophilic anion where it acts as a proton shunt (9), dissipating $\mathrm{pH}$ gradients across a range of host cellular-organelle membranes, including mitochondria, endosomes and lysosomes, and even the Golgi. In the endosome it blocks entry of viral RNA (17) by not allowing the endosome $\mathrm{pH}$ to acidify, thereby preventing the acidic-pH-dependent conformational change required by the coronavirus spike protein to fuse with the endosome membrane $(26,27)$ ). Similarly, as shown for other viruses it could potentially inhibit coronavirus assembly in the Golgi (28). One of its main effects though is in the inner mitochondrial membrane. Here it dissipates the $\mathrm{pH}$ gradient required to drive ATP synthase (9). This, in turn, reduces the cell's production of its main energy molecule, adenosine triphosphate (ATP). Niclosamide's oxidative phosphorylation effects are therefore enabled by a reduction in ATP that is upstream of many key cellular processes including transcription and translation of viral RNA (29). ATP is also a substrate for the multi-subunit enzyme RNA polymerase that adds ATP and the other ribonucleotides to a growing RNA strand. Furthermore, as described by Zimmerman et al., (30), ATP serves as a cofactor for signal transduction reactions using a variety of kinases as well as adenyl cyclase. Hence, the production and presence of ATP in every cell is essential to their functioning on multiple levels. Normally, cellular ATP concentration is maintained in the range of 1 to $10 \mathrm{mmol} / \mathrm{L}$. However, titrating with niclosamide reduces the ATP content as readily measured using assays for cell "viability", such as Cell Titer Glo (31). In vaccinia poxvirus, virus production actually requires increased amounts of ATP $(32,33)$, and so, if this holds for coronavirus, niclosamide could provide an even greater inhibitory effect.

Thus, niclosamide, at relatively low concentrations, in only the tens of micromolar range, basically turns down the dimmer switch on the cell's energy production while still preserving reversible viability (34). As such, niclosamide can inhibit virus replication as 
demonstrated in Vero 6 cells at only $1 \mu \mathrm{M}(8)$ and has also been shown to have similar anti-replicative efficacy in cultured Calu-3 lung cells, at $2 \mu \mathrm{M}$ (35). Data is still needed on actual airway epithelial cells which is essential to show for its potential use in COVID prevention and early treatment.

\section{The Need for New Formulations}

Because of these quite dramatic and potentially efficacious effects, and the urgency of the COVID19 pandemic, in addition to relatively unsuccessful attempts to repurpose the original oral tablets, there have been efforts to develop new formulations and routes of administration for niclosamide, to validate them in preclinical studies, and clinically test niclosamide for COVID-19 (see this reference to clincaltrials.gov (36)). These trials include new intramuscular injections by Daewoong Pharmaceuticals of South Korea (37), and a nasal spray and inhalant as a spray-dried-lysozyme particulate of micronized-niclosamide (3), that has entered a 1,500 high-risk kidney patient study in the UK with Union Therapeutics in Denmark (38).

However, regarding the nasal spray, microparticles are not expected to penetrate the mucin barrier that protects the respiratory epithelial cells (39-41), and so this now motivates a deeper physico-chemical evaluation of niclosamide and especially how to increase its concentration in solution using simple buffers to access the epithelial cells. The goal of this study is therefore to optimize the bioavailability of niclosamide as a mucinpenetrating solution and prophylactically saturate the first layer of epithelial cells that are the targets for SARS-CoV-2 viral infection (42). It is also to provide a similar throat spray to reduce viral load and help treat early infection.

\section{The Main Issue is Optimization of Drug Bioavailability}

The main issue for any formulation of niclosamide (and indeed other BCS Class II and IV low water-solubility drugs) is how to optimize local bioavailability. This is especially important in the nose and throat. There are two parts to this: the intrinsic low solubility of niclosamide in aqueous solution compared to the concentration required for efficacy; and the fact that the more traditional choice for nasal sprays of microparticles cannot penetrate the mucin (39-41).

While its efficacy at inhibiting viral replication has been measured in Calu- 3 cells to be $2 \mu \mathrm{M}-3 \mu \mathrm{M}(4,35)$, the amount of niclosamide that can be dissolved in aqueous solution at neutral or lower $\mathrm{pH}$, as encountered in the nasal pharynx (43), is of the same order, or perhaps slightly less, i.e., $\sim 1 \mu \mathrm{M}-2 \mu \mathrm{M},((2)$ and as measured here, especially for the low solubility monohydrate). Because of this low aqueous solubility, one might imagine that intranasal microparticle delivery would increase the amount delivered. However, the mucosa is designed to protect the nasal epithelial cells and prevent the permeation of particles with a size greater than a few hundred nanometers (41). As a result, any microparticle formulation cannot deliver molecular drug directly to the epithelial cells where initial infection takes place because of the protective mucin barrier that is produced by, and covers, these cells (41). This means that any drug microparticle suspension can only provide the fraction of drug that is soluble in aqueous solution in any delivered suspension. Nevertheless, this may still show some efficacy depending on the drug solubility-to-efficacy ratio.

A good example is Flonase (44) and Nasonex $(45,46)$ (see also Supplemental Information S1.2). Solubilities of their APIs, fluticasone propionate and mometasone furoate, are reported to be $21 \mu \mathrm{M}$ and $23 \mu \mathrm{M}$, respectively (Drugbank, ALOGPS). By comparison, the relative trans-activation potency $\left(\mathrm{EC}_{50}\right)$ for fluticasone propionate is $0.320 \pm 0.04 \mathrm{nmol} / \mathrm{L}$ and that for mometasone furoate is even less at $0.069 \pm 0.021 \mathrm{nmol} / \mathrm{L}$. That is, the amount of drug required to induce efficacy is 6,000 and 30,000 times greater in bioavailable aqueous concentration of the drugs compared to their nanomolar efficacy. However, for the low solubility niclosamide, this solubility-to-efficacy ratio is 1 or less (see also Supplemental Information S1.3). Again, microparticle niclosamide formulations may show some efficacy but are therefore clearly not optimal. There is therefore a need for a more bioavailable solution.

\section{Meeting the Challenge with a Simple Solution}

It is here that we have focused on what it takes to provide a mucus-penetrating niclosamide solution rather than microparticulate material that is simply trapped at the mucus surface, and then excreted as the mucin is replaced (every 15-20 min (47)). We have found a way to increase the solubility of niclosamide and therefore its bioavailability to infectable and infected epithelial cells just by slightly increasing the $\mathrm{pH}$ in simple aqueous buffered solutions (48). This now optimizes the delivery of niclosamide in a low dose prophylactic nasal spray and as a higher dose early treatment throat spray.

As described in Results and Discussion, at $\mathrm{pH}$ 8.0, a $20 \mu \mathrm{M}$ niclosamide nasal solution is within the natural $\mathrm{pH}$ range for the nasopharynx and, at $\mathrm{pH}$ 9.2, a higher concentration $300 \mu \mathrm{M}$ throat spray is at a tolerable $\mathrm{pH}$ for the oropharynx. These niclosamide solution concentrations are $\sim 10 \times$ to $\sim 150 \times$ above the $100 \%$ in vitro 
inhibition of SARS-CoV-2 viral replication as measured to date in Vero 6 and Calu-3 cells of $1 \mu \mathrm{M}-3 \mu \mathrm{M}$ (4, $35,49)$. Also, preliminary studies by Kim (34) in airway epithelial cells, show that at an $\mathrm{EC}_{50}$ of $20 \mu \mathrm{M}$ to $30 \mu \mathrm{M}$ niclosamide gives the necessary reductions in cell ATP, and that this lowered cell viability is reversible and does not result in any cell death. While the vaccine is highly protective against hospitalization and severe disease, break through infections are now occurring, and virus is being spread by the asymptomatic-vaccinated requiring new mask mandates, that are linked to the delta, and potentially other new variants $(50,51)$. Prophylactic nasal and early treatment throat sprays could "put the virus in lockdown" or not even let the viral RNA into the nasal epithelial cells. As such, they could be a huge boost in the control of viral load and reduce development of infection for vaccinated and especially unvaccinated and spreaders.

The envisioned final products are solutions of niclosamide made up in $\mathrm{pH}$ buffer and available in $10 \mathrm{~mL}$ doses in $15 \mathrm{~mL}$ nasal and throat spray bottles. The low dose $20 \mu \mathrm{M}$ niclosamide solution at $\mathrm{pH} 7.9$ can be sprayed intranasally and the higher dose $300 \mu \mathrm{M}$ at pH 9.2 sprayed to the back of the throat.

\section{Bottom Line}

With the coronavirus pandemic still raging, more contagious (delta and omicron) variants already in circulation (1), according to OurWorldinData.org, (52) currently in the USA the number of people fully vaccinated $(11 / 07 / 2021)$ is $192.4 \mathrm{M}$, and so still only $57.2 \%$ of the total population. Across the world, the average fully vaccinated is now $(40.6 \%, 3.13$ billion/7.71) billion. However, in Africa, fully vaccinated is only $6.2 \%$ and only $4.2 \%$ of people in low-income countries have received at least one dose. Thus, there is still an urgent need for additional mitigation efforts to support current and potential vaccinations. For unvaccinated (including children and babies where COVID cases are now rising (53)), anti-mask, and anti-vaccination populations, an effective nasal prophylactic and early treatment throat spray would serve as protection behind the mask and enhance mitigation efforts.

Thus, while vaccines and antibody treatments are certainly effective, they are designed to work only after infection has taken place, i.e., they do not prevent infection, as we are now seeing with breakthrough infections and viral spreading even for vaccinated persons (50, 51). Vaccines are also limited to a particular virus, a particular viral protein, and eventually need to be boosted or redesigned for an emerging viral variant. In contrast, Niclosamide is a universal host cell modulator and so is specific for every virally infected or infectable cell. As postulated by Laise et al. (24), "Targeting host cell mechanisms may have more universal and longer-term value because the same host factors may be required by multiple, potentially unrelated viral species and because host target proteins mutate far less rapidly than viral proteins, thereby limiting emergence of drug resistance". We concur; and would add that lipid bilayer membranes mutate even less.

\section{RESEARCH DESIGN}

We have much previous experience working with and preparing niclosamide for drug delivery, including as a niclosamide prodrug therapeutic for anti-cancer applications $(54,55)$. Following publication of the bioRxiv preprint by Jeon et al. in March 2020 (8) (now peerreviewed (49)), that demonstrated anti-viral activity of niclosamide against SARS-CoV-2, our efforts were refocused on ways to increase the amount of niclosamide in sprayable nasal and throat suspensions (48). A description of the initial thinking that went into the work and that led to the solution formulation is given in the Supplemental Information S1. It is an interesting account that, along with this Research Design section, should help to inform what went into the research and development. It includes: more on the case of Nansonex and Flonase; that Niclosamide's solubility at physiologic $\mathrm{pH}$ is actually in the same range as its efficacy; could Niclosamide be encapsulated or stabilized as nanoparticles? and, given the "solution" to the problem, how much niclosamide is actually sprayed per dose?

In preparation for designing and making any drug delivery system, it is also important to carry out a preformulation drug characterization (see Supplemental Information S2). Such analyses are focused on the basic molecular properties of niclosamide including its $\mathrm{pKa}$, the intrinsic solubility of its protonated $\left(\mathrm{S}_{\mathrm{o}}\right)$ and deprotonated $\left(\mathrm{S}_{-\mathrm{ve}}\right)$ forms, the resulting $\mathrm{pH}$-dependent amount of niclosamide in solution, and its octanol water partition coefficients $(\operatorname{LogP}$, and the $\mathrm{pH}$-dependent $\log \mathrm{D})$. These all underly the ability of niclosamide (and especially dependent on the polymorph supplied), to partition into the various lipid bilayer membranes (hence $\log \mathrm{B}$ ) and exert its proton shunt effect.

\section{Overall Goal and Aims}

The overall scientific goal of this study was to explore the expected $\mathrm{pH}$-dependence of Niclosamide implied by the literature and calculation databases for its $\mathrm{pKa}$ of $\sim 6-7(9,56-58)$. This indicated that the amount of 
niclosamide in aqueous solution should increase with increasing $\mathrm{pH}$ above neutral. Experiments were therefore carried out to evaluate this $\mathrm{pH}$ dependence and compare it to theory for a sourced niclosamide from a chemical supplier, (AK Sci, CA). The initial aims therefore were to measure the equilibrium $\mathrm{pH}$ dependence of niclosamide (AK Sci) in a series of buffered solutions (nominally pHs $4-9.5$ ) and its rate of dissolution in the same buffer series. However, on occasion, supernatant solutions that had attained equilibrium with the slight excess of undissolved solid material, showed a reduction in concentration, indicating that some samples were moving towards a lower solubility solid form. A second series of experiments were therefore deigned to explore these conversions that are already known for niclosamide $(2,59-61)$ but have not been investigated as a function of $\mathrm{pH}$. Equilibrium supernatant concentrations and dissolution rates for niclosamide from a second supplier, (Sigma), were therefore investigated, as were precipitated samples and specially prepared water, acetone and ethanol cosolvates. As reported in Results, while the Sigma sample initially produced similar dissolution and supernatant concentrations as the AKSci material, when left stirring overnight, it converted to a lower solubility polymorph. When precipitated at each $\mathrm{pH}$, there was still a $\mathrm{pH}$ dependence to the amount of niclosamide in solution but now at a much lower level than the as-supplied AK Sci niclosamide powder. Niclosamide precipitated from supersaturated solution and the cosolvates also provided a lower amount of niclosamide in supernatant solution.

\section{Specific Aims}

Experimentation therefore focused on five main specific aims (SAs).

\section{SA I. To Measure the Equilibrium Dissolution of Niclosamide versus $\mathrm{pH}$ from a Commercial Supplier (AK Sci)}

As predicted by Henderson Hasselbalch and precipitation $\mathrm{pH}$ models, the first task was to confirm that the amount of niclosamide in buffered solution increased with increasing $\mathrm{pH}$ over the nominal $\mathrm{pH}$ range 4 to 9.5. Small excesses of powdered niclosamide from a commercial supplier (AK Sci, CA) were equilibrated in $\mathrm{pH}$ buffers in stirred vials and the filtered supernatant niclosamide concentration was measured using a calibrated UV/Vis protocol. Optical microscopy was used to obtain images of the particle morphologies at each $\mathrm{pH}$.
Sa 2. To Measure Dissolution Rates of AK Sci and Sigma Niclosamides as a Function of $\mathrm{pH}$ Including a New Methodology for Measuring the Supernatant Concentration In Situ

In a series of dissolution measurements, a new methodology was established for measuring the supernatant concentration of niclosamide in situ using UV/ Vis absorption of stirred niclosamide solutions equilibrated with supplied powdered niclosamide. This new sampling technique utilized a nanodrop UV/Vis spectrophotometer for detection of niclosamide at $333 \mathrm{~nm}$ and $377 \mathrm{~nm}$ using only $2 \mu \mathrm{L}$ samples drawn directly from the stirred suspensions. It allowed the measurement of initial dissolution rates and full dissolution curves in situ as the drug was dissolving versus time. Dissolution time periods were $60 \mathrm{~min}$ and longer for a series of $\mathrm{pH}$ solutions using the AK Sci and Sigma niclosamides, an optimized mixing protocol was also developed.

\section{SA3. To Precipitate Niclosamide from Supersaturated Solution and Measure its Equilibrium Supernatant Concentrations at the Same $\mathrm{pHs}$}

When precipitated from supersaturated solution the formed microcrystals are expected to take on their "natural" morphology associated with nucleation and growth at that $\mathrm{pH}$. Using the solvent exchange technique, it was possible to obtain such precipitated material and measure their equilibrium niclosamide solution concentrations corresponding to precipitation at each $\mathrm{pH}$. Optical microscopy again allowed images to be obtained of the particle morphologies at each $\mathrm{pH}$.

\section{SA 4. To Evaluate Dissolution Rates and Equilibrium Niclosamide Concentrations for Niclosamide- "Cosolvates" (from Water, Acetone and Ethanol)}

The above studies led to a series of experiments that sought to provide additional information as to the nature of the commercially available niclosamide material that dissolved so readily (AK Sci and Sigma), but where, in one case (Sigma) it converted to a lower solubility form, while in the other (AK Sci) was mostly stable at high concentration for days in equilibrium with its original powder. Niclosamide was therefore precipitated into water to obtain the fully hydrated form or was recrystallized from acetone and ethanol, dried as powders, and then dissolved to equilibrium in $\mathrm{pH} 9.3$ buffer to determine their dissolution profiles, final niclosamide concentrations, and particle morphology, again by optical microscopy. Note: a similar protocol was used to make them 
as reported by van Tonder et al., (61). Subsequent characterization by (Burley et al. Nottingham (62)) using Raman spectroscopy/microscopy, infra-red spectroscopy, X-ray diffraction (powder and single crystal), thermal methods, will show if they are truly cosolvates.

\section{SA 5. To Measure Equilibrium Supernatant Concentrations of Sigma Niclosamide as a Function of $\mathrm{pH}$ ( $3 \mathrm{~h}$ and Overnight Stirring)}

Finally, as was done for the AKSci material, SA 5 was to confirm that the amount of niclosamide in buffer solution from a different supplier, (Sigma, MO), also increased with increasing $\mathrm{pH}$. Here a series of samples were made over the $\mathrm{pH}$ range 3.6 to 9.5. Equilibration was again measured for small excesses $(1 \mathrm{mM})$ of powdered niclosamide in stirred vials equilibrating for $3 \mathrm{~h}$ and also after $24 \mathrm{~h}$, measuring the supernatant concentration using a calibrated cuvette $\mathrm{UV} / \mathrm{Vis}$ protocol. The new equilibrium concentrations were measured after filtering through a $0.22 \mu \mathrm{m}$ filter. Optical microscope images were obtained of particle morphologies at each $\mathrm{pH}$. What was discovered here was that this form of niclosamide converted to the low solubility polymorph after overnight stirring because the starting material already contained some of this polymorph.

\section{EXPERIMENTAL}

\section{Materials}

Niclosamide was from AK Sci (CA) (Lot No. 90402H, listed as, at least $98 \%$ pure by HPLC) and from Sigma, (N3510); Water was deionized and filtered through $0.22 \mu \mathrm{m}$ filters. TRIZMA buffer was from Supelco and comprised: Trizma Base, $99.8+\%$, reagent grade Tris(hydroxymethyl)-aminomethane (HOCH2)3CNH2, Mol Wt $121.14 \mathrm{~g} / \mathrm{mol}$, white crystalline powder; Trizma $\mathrm{HCl}, 99+\%$, reagent grade (Tris[hydroxymethyl] aminomethane hydrochloride), (HOCH2)3CNH2•HCI Mol Wt $157.60 \mathrm{~g} / \mathrm{mol}$, white crystalline powder. Ethanol KOPTEC $200 \%$ proof and acetone BDH 1101 were from VWR. pH buffers were made using the TRIZMA $\mathrm{HCl}$ and TRIZMA base buffer system (Supelco). From a 1L stock solution at $\mathrm{pH}$, a series of $100 \mathrm{~mL}$ buffer solutions in $250 \mathrm{~mL}$ glass screw capped bottles were made nominally at pHs of 4.0, 7.0, 7.5, 8.0, 8.25, 8.5, 8.75, 9.0, 9.3 and 9.5 At the highest TRIZMA base-concentrations it was possible to exceed $\mathrm{pH} 9$ and extend the range to $\mathrm{pH} 9.5$.
The $\mathrm{pH}$ solutions were made by adding to the stirred pH 9.5 solution suitably small amounts of $2 \mathrm{M} \mathrm{HCl}$ using a pipettor and fine-tuned with $0.1 \mathrm{M}$ to achieve the lower $\mathrm{pHs}$ or by adding small amounts of Trizma base to re-raise the $\mathrm{pH}$. $\mathrm{pHs}$ were measured using a Mettler SevenEasy ${ }^{\mathrm{TM}} \mathrm{pH}$ Meter S20, calibrated prior to any measurements using standard (VWR) buffers of $\mathrm{pH} \mathrm{4,} 7$ and 10. Nominal $\mathrm{pH} 4$ buffer was made using sodium citrate-citric acid buffer.

AKSci niclosamide purity was measured by HPLC-UV Chromatogram for Niclosamide in $\mathrm{MeOH}$ and was found to be $98.4 \%$ pure based on \%-area, and so agreed with the AKSci data.

Niclosamide was also found to be stable in a Forced Degradation test, where a solution of niclosamide was prepared at $100 \mu \mathrm{g} / \mathrm{mL}$ in $1 \mathrm{~N} \mathrm{NaOH}$, stored at room temperature, and periodically analyzed by LCMS over a 5-day period. Degradation to its breakdown products of 2-chloro-4-nitroaniline and 5-chloro-2-hydroxybenzoic acid were observed at this high $\mathrm{pH}$. However, at neutral $\mathrm{pH}$ and $\mathrm{pH} 9$, the solutions were stable as niclosamide for at least 16 days of measurement.

\section{Methods}

\section{Solvent Exchange Technique for Making Calibration Standards} and Supersaturated Solutions

The solvent exchange technique, normally used to make nanoparticles (54), can readily be used to make small volumes of niclosamide solutions. The procedure is to simply inject a relatively concentrated ethanolic niclosamide solution into an excess of anti-solvent, i.e., the stirred buffer. Figure 1 shows the eVol syringe, $20 \mathrm{~mL}$ scintillation vial, and magnetic stirrer set up.

Basically, the technique involves diluting a concentrated ethanolic niclosamide solution and exchanging the good solvent (ethanol) for the anti-solvent (aqueous buffer). This is done in such a way that the final concentration does not exceed the solubility limit of niclosamide in the buffer at that $\mathrm{pH}$. As a result, there is no precipitation of niclosamide (this was checked via Dynamic Light Scattering, (DLS)). As shown in Fig. 1, an eVol syringe (Trajan Scientific and Medical. Trajan Scientific Australia Pty Ltd) is clamped securely in a commercially available drill press (Yeezugo, Guangzhou, China). This mounting allows for repeatable and accurate positioning of the syringe needle tip in the stirred solution, -a feature that is essential for injection of the solution into the most effectively stirred volume of the solution in the vial. While less of an issue when making solutions, repeatable and consistent injection 
Fig. I eVol syringe mounted in drill press, with $20 \mathrm{~mL}$ scintillation vial and magnetic stirrer set up, showing the simple procedure for introducing microliters of ethanolic niclosamide into the stirred vial.

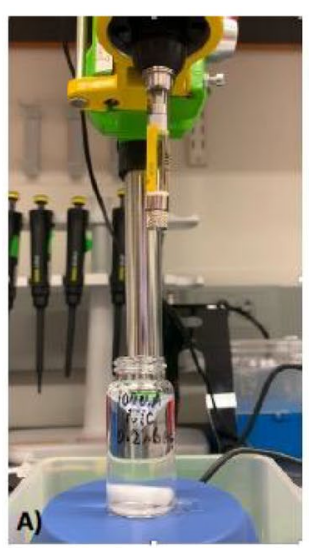

Buffer solution stirring, syringe needle in ready position

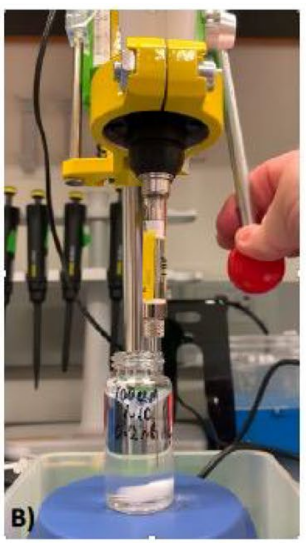

Syringe needle positioned in stirred buffer solution

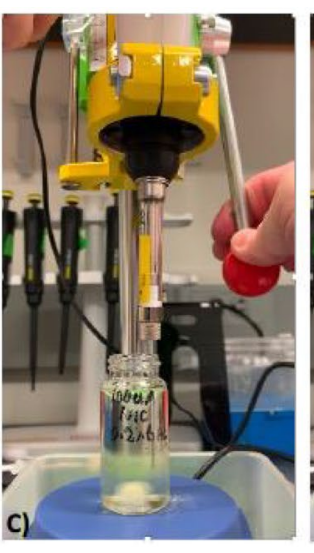

Syringe activated and desired volume of ethanolic niclosamide injected into buffer forming yellow clear solution

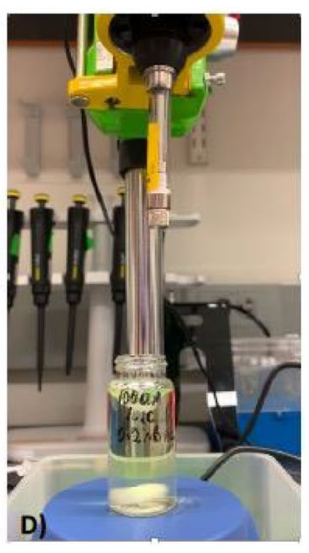

Syringe needle withdrawn, solution ready for further characterization (e.g., DLS to ensure no particles and UV/Vis for concentration) speed and mixing environment is critical for supersaturation precipitation since the vortex is not the most efficiently stirred part of the system (63) and particle nucleation is very sensitive to the mixing environment (54).

The protocol for making the standards or creating supersaturated solution to precipitate material is as follows:

Withdraw $12 \mathrm{ml}$ of the buffer solution into a $30 \mathrm{~mL}$ BD syringe. Insert and luer-lock a $0.22 \mu u m$ filter to the syringe and prime the filter by expelling $\sim 2 \mathrm{mLs}$ of solution into a waste beaker. Add $9.9 \mathrm{mLs}$ of the solution from the syringe through the filter into the $20 \mathrm{~mL}$ scintillation vial. Load the eVol syringe with $100 \mu \mathrm{L}$ of a desired niclosamide solution in ethanol that has also been filtered through a $0.22 \mu \mathrm{m}$ filter to remove any insoluble residual particulate material in the supplied niclosamide powder. Add an ethanol-cleaned, small magnetic stir bar to the vial and place the vial on a magnetic stirrer as shown in the photographic images in Fig. 1. Turn on the magnetic stirrer to create a small vortex (Fig. 1A). Bring down the syringe needle and position it over to the side of the vortex (Fig. 1B) i.e., for better mixing the syringe tip needs to be close to the ends of the stir bar. For the stock standard, inject the $100 \mu \mathrm{L}$ of $30 \mathrm{mM}$ ethanolic Niclosamide into the $9.9 \mathrm{mLs}$ of stirred $\mathrm{pH} 9.3$ buffered solution at moderate injection rate (Fig. $1 C$ ). Within 1 to $2 \mathrm{~s}$ of finishing the injection, turn off the stir motor, raise the needle (Fig. 1D) and remove the vial. This method makes $10 \mathrm{mLs}$ of $300 \mu \mathrm{M}$ Niclosamide including $1 \%$ residual ethanol. For precipitation of niclosamide from supersaturated solution the desired concentration of ethanolic niclosamide was used to provide a final niclosamide concentration that was above the solubility of niclosamide in solution at a particular $\mathrm{pH}$. For more details on calibration standards including results, see Supplemental Information S3.

\section{Equilibrium Dissolution of AK Sci Niclosamide versus $p H$}

A series of equilibrated niclosamide solutions were prepared at each nominal $\mathrm{pH}$ from 4 to 9.5 (4, 7, 7.5, $8,8.25,8.5,8.75,9,9.3,9.5)$ by dissolving the AK Sci niclosamide at concentrations that were in excess of its expected solubility, i.e., $100 \mu \mathrm{M}(0.33 \mathrm{mg} / 10 \mathrm{mLs})$ for $\mathrm{pH} 4,7,7.5$ and $8 ; 300 \mu \mathrm{M}(1 \mathrm{mg} / 10 \mathrm{mLs})$ for $\mathrm{pH}$ $8.25,8.5$ and 8.75$)$ and $1 \mathrm{mM}(3.3 \mathrm{mg} / 10 \mathrm{mLs})$ for $\mathrm{pH}$ $9,9.3$ and 9.5 . As can be appreciated, again, very small amounts were required, $(\sim 0.33 \mathrm{mg}-3.3 \mathrm{mgs})$. Niclosamide, as obtained directly from the supplier (AK Sci) was weighed into a weigh boat and added to $10 \mathrm{mLs}$ of each buffer solution in $20 \mathrm{~mL}$ screw top scintillation vials.

The solutions and undissolved particles in the vials where then briefly shaken by hand to wet and immerse the hydrophobic niclosamide powder and stirred by magnetic stirrers. UV-Vis measurements by cuvette were made (UV5 nano, Mettler Toledo) a few hours after making and stirring, and after 1-8 days to ensure equilibrium. Absorbance was compared to a standard calibration curve (given in Supplemental Information S3). It is important to show this because the $\mathrm{pH}$-dependent spectra are actually new.

Since some suspensions were visibly cloudy due to the excess undissolved material. In order to avoid sampling particles that could interfere with the UV/Vis cuvette measurement, $0.5 \mathrm{mLs}$ of the supernatants were 
taken, spun down by Eppendorf-centrifugation (10 min at $15,000 \mathrm{G}$ ) to remove any suspended particles, and analyzed by UV/Vis spectrophotometry. Full spectra were recorded and the absorbance at the $333 \mathrm{~nm}$ peak was compared to the calibration in order to determine the niclosamide concentrations of the supernatant. Their final $\mathrm{pH}$ was remeasured on a Mettler Toledo $\mathrm{pH}$ meter.

For the UV/Vis measurements, each buffer was used as its own blank, and at least 5 individual samples were taken for each buffer and averaged to establish the "blank baseline" which was usually between 0.000 and 0.003 absorption values. These values were subtracted from the niclosamide measurements. This subtraction was particularly important for the very low niclosamide concentrations at pHs 3.66 and 7, where the signal was very close to, but still distinguishable from, the usual noise of the instrument.

\section{Rates of Dissolution for AK Sci and Sigma Niclosamide Powders}

The dissolution of niclosamide powder was measured in a timed dissolution study using a nanodrop UV/Vis spectrophotometer (Thermo Fischer nanodrop and Mettler Toledo, UV5nano). In order to try and standardized the particle size and therefore surface area of the niclosamide particles, the as-received powder was ground with a mortar and pestle. This was actually done for all samples, (from Sigma, the water-precipitated niclosamide, and the ethanol and acetone cosolvates). Appropriate amounts of AK Sci or Sigma niclosamide powder, as in the above equilibration studies were weighed into dry $1.5 \mathrm{~mL}$ Eppendorf tubes and capped. Since, as shown in results, final concentrations of niclosamide for $\mathrm{pH} 7$ to 8.25 were still relatively low, only $\mathrm{pH} 8.5,8.75,9,9.3$ and 9.5 were tested. $10 \mathrm{mLs}$ of each buffer were aliquoted from a $10 \mathrm{~mL}$ BD syringe fitted with a $0.22 \mu \mathrm{m}$ filter into $20 \mathrm{~mL}$ scintillation vials, and a cleaned magnetic stirbar was added to the vial. At time $(t)=0$, the contents of one of the Eppendorfs was rapidly emptied into the buffered solution vial. The vial and solution were quickly shaken by hand in order to wet and immerse the hydrophobic powder into suspension, and the vial was placed on the magnetic stirrer. $2 \mu \mathrm{L}$ samples for $\mathrm{UV} / \mathrm{Vis}$ measurement on the nanodrop spectrophotometer were taken by a 2-20 $\mathrm{LL}$ pipettor at regular time intervals, e.g., every $30 \mathrm{~s}$ up to $7 \mathrm{~min}$, every minute from $8-16 \mathrm{~min}$, every $2 \mathrm{~min}$ from 18-30 min and then every $5 \mathrm{~min}$ from $30-60 \mathrm{~min}$. Thereafter, samples were taken at 90,120 , and $180 \mathrm{~min}$, as well as the next day.

Thus, here, a new technique for measuring drug dissolution was developed. Rather than having to employ large volumes of suspension and a series of tubes, connectors, pumps, filters, and stirred cuvettes, $2 \mu \mathrm{L}$ samples were taken directly from the $10 \mathrm{mLs}$ of suspension in a stirred $20 \mathrm{~mL}$ vial. This allowed direct in situ measurements to be made of the dissolution of the various niclosamide samples as a function of time at each $\mathrm{pH}(8.5-9.5)$. This gave their initial rates of dissolution including more optimized mixing conditions for the supplied material. By utilizing $2 \mu \mathrm{L}$ sampling, on a limited excess of powdered material in the stirred supernatant and making measurements on the nanodrop spectrophotometer, it was very rare for the $2 \mu \mathrm{L}$ volume to include a powder particle. In any event, if this happened, the nanodrop software identified it as perhaps a bubble or error due to changed path length, and the measurement could be excluded and repeated.

\section{Supersaturated Solution Precipitation of Niclosamide: Solubilities at Each pH and Corresponding "Natural" Morphologies}

This next experiment was carried out in order to determine the amounts of niclosamide in solution corresponding to what are expected to be the "natural" morphologies of niclosamide when precipitated at each $\mathrm{pH}$. Using a series of stock solutions in ethanol, final supersaturated concentrations of niclosamide were created in each $\mathrm{pH}$ buffer in slightly excess amounts, i.e., just enough to generate precipitated material given that time was required for the stochastic nucleation and precipitation to occur. This excess, kinetically soluble, niclosamide was mixed into the buffers using the solvent injection technique. For example, $33 \mu \mathrm{L}$ of $30 \mathrm{mM}$ into $10 \mathrm{mls}$ of buffer gave supersaturated solutions of $100 \mu \mathrm{M}$ for $\mathrm{pH} 3.66,7,7.5,8,8.25 ; 100 \mu \mathrm{L}$ of $30 \mathrm{mM}$ gave a $500 \mu \mathrm{M}$ supersaturated solution for pH 8.5 and 8.75 ; and $333 \mu \mathrm{L}$ of $30 \mathrm{mM}$ gave a $1 \mathrm{mM}$ supersaturated solution for $\mathrm{pH} 9.0,9.3$ and 9.5. The solutions were again stirred until precipitation was observed, often overnight and sometimes longer. In the case where no precipitate was seen in the $\mathrm{pH} 9.5$ solution after 1-2 days stirring, additional volumes of $30 \mathrm{mM}$ ethanolic niclosamide were added and the solution continued stirring until a precipitate was obtained. For this $\mathrm{pH} 9.5$ sample, solution concentrations had to be increased to $3 \mathrm{mM}$ supersaturation and even then, the kinetic solution was stable for at least a few hrs at $3 \mathrm{mM}$ niclosamide. The precipitated suspensions were examined by optical microscopy using bright field optics, Köhler illumination, and a $40 \times$ objective to obtain micrograph images of the precipitates. $0.5 \mathrm{mLs}$ of supernatants were centrifuged in $1.5 \mathrm{ml}$ Eppendorf tubes for $10 \mathrm{~min}$ at $15,000 \mathrm{G}$ to obtain clear supernatant solutions that were in equilibrium with 
the various precipitated morphologies. UV/Vis spectra and $333 \mathrm{~nm}$ intensities were again obtained by the nanodrop spectrophotometer.

\section{Dissolution of Niclosamide Solvates}

To start to evaluate the various expected polymorphs and to try and obtain a morphology like the purchased materials (of unknown processing), three niclosamide (AK Sci) samples were made i.e., the "water-hydrate" precipitated from ethanol into excess water, and two recrystallized samples from acetone and ethanol presumed to be "cosolvates" because the method of making followed that by van Tonder et al. $(2,59,61)$ for their various cosolvates that were evaluated by x-ray diffraction, IR spectra, and calorimetry. These were made and evaluated as follows.

Water precipitate Niclosamide (AK Sci) was precipitated from supersaturated solution by solvent exchange into excess deionized water by injecting $400 \mu \mathrm{L}$ of ethanolic $25 \mathrm{mM}$ niclosamide solution into $10 \mathrm{mLs}$ of stirred pH 9.3 buffer, (3.8\% residual ethanol) i.e., much as might be done after synthesizing the niclosamide dissolving in an ethanol solvent and recovering by precipitation into excess water. The niclosamide precipitated immediately as its usual, visible by eye, white precipitate (see image in Figure S1 Supplemental Information). It was filtered on a sintered glass filter, washed three times with deionized water, and dried. The material was ground by mortar and pestle ready for the dissolution test and viewed microscopically to evaluate its crystal morphology.

Niclosamide Recrystallized from Acetone and Ethanol Niclosamide was recrystallized from acetone and ethanol by dissolving excess niclosamide into the solvent, gently warming in a fume hood to dissolve excess niclosamide, warmed to evaporate-off $\sim 50 \%$ of the solvent, that were then allowed to cool under stirring. The recrystallized niclosamide solvates were filtered and dried. Each material was also viewed microscopically to evaluate their crystal morphologies.

Dissolution of the solvates As with the AK Sci and Sigma materials, $3.3 \mathrm{mg}$ of niclosamide from each of the hydrate and cosolvates was weighed and added to stirred pH 9.3 buffer to give a nominal $1 \mathrm{mM}$ niclosamide in suspension ready to dissolve. $2 \mu \mathrm{L}$ aliquots were taken directly from the stirred solution in the $20 \mathrm{~mL}$ scintillation vial at appropriate time intervals (as above) and absorbance at $333 \mathrm{~nm}$ was measured by nanodrop spectrophotometer.

\section{Equilibration of Sigma Niclosamide and its conversion to the Low Solubility Form}

A final experiment evaluated equilibration of niclosamide from a second supplier, (Sigma), over the $\mathrm{pH}$ range 7 to 9.5. This powder was much lighter in color, a creamy yellow, as opposed to the browner yellow of the AK Sci product. As before, excess niclosamide was weighed and added to individual $10 \mathrm{mLs}$ of buffer solution in $20 \mathrm{~mL}$ vials to give an equivalent of $100 \mu \mathrm{M}$ $(0.33 \mathrm{mg})$ for $\mathrm{pHs} 7,7.5$ and $8.0 ; 200 \mu \mathrm{M}(0.65 \mathrm{mg})$ for pHs 8.25 and $8.5,500 \mu \mathrm{M}(1.65 \mathrm{mg})$ for $\mathrm{pHs} 8.5$ and 9.0 and $1 \mathrm{mM}(3.3 \mathrm{mg}$ ) for pHs 9.3 and 9.5 . As above, the vial and solution were quickly shaken by hand in order to wet and immerse the hydrophobic powder into suspension, and the vial was placed on the magnetic stirrer. The suspensions were stirred by magnetic stirbar for three hours. At this point $0.5 \mathrm{mls}$ of each were centrifuged at $15,000 \mathrm{G}$ for $10 \mathrm{~min}$ and the supernatant niclosamide concentration was measured by UV/Vis (cuvette) spectrophotometer. At least 5 samples were taken to give the average niclosamide absorbance. Each $\mathrm{pH}$ buffer was used to blank the measurements and the blank values were again subtracted from the niclosamide absorbance values.

Samples were left stirring overnight. Again, $0.5 \mathrm{mls}$ of each were centrifuged at $15,000 \mathrm{G}$ for $10 \mathrm{~min}$ and the

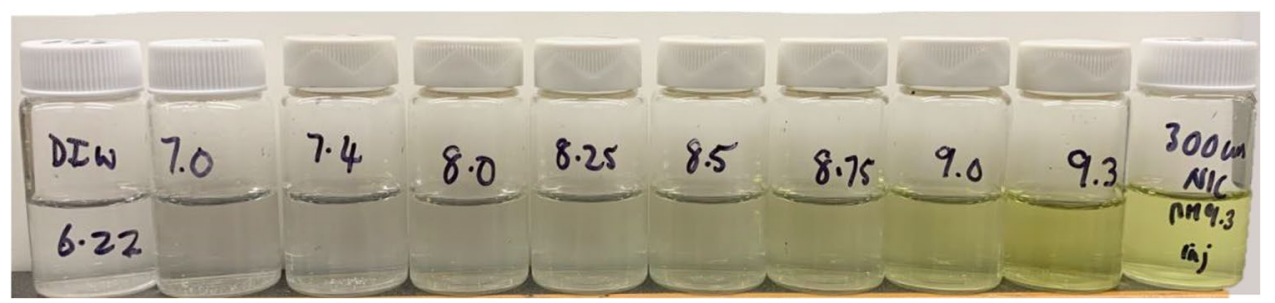

Fig. $210 \mathrm{~mL}$ samples of excess niclosamide dissolved in $\mathrm{pH}$ buffers (nominally $7.0-9.3$ ). Also shown is a deionized water sample nominally $\mathrm{pH} 6.22$ (far left,) and a 300uM Niclosamide in a pH 9.3 standard (far right), made by solvent injection (measured at $30 \mathrm{I} \mu \mathrm{M} \pm 5 \mu \mathrm{M}$ ). The image shows the increasing "yellowness" characteristic of niclosamide in solution. Excess powdered AK Sci niclosamide is seen at the bottom of each vial. (NOTE: While these images are of equilibrated samples, in the dissolution experiments, when stirred into the supernatant, there was such a small amount that it was rarely included in the $2 \mu \mathrm{L}$ sampling. 
Fig. 3 Equilibrated supernatant concentrations for dissolution of powdered niclosamide (from AK Sci) added in excess to each $\mathrm{pH}$ buffer at $20^{\circ} \mathrm{C}$. A Supernatant $\mathrm{pH}$ versus the Supernatant Niclosamide concentration [Nic] $(\mu \mathrm{M})$ measured by UV5 Nano (Mettler Toledo) UV/Vis and compared to the $\mathrm{pHp}$ curve, Eqn in text (see also Eq. 4 in Supplemental Information, S2.3. The precipitation $\mathrm{pH}(\mathrm{pH} \mathrm{p}))$. Plotting this data against the $\mathrm{pH}$ p predictions for a measured intrinsic solubility for AKSci niclosamide Sol of $2.53 \mu \mathrm{M}$, gives a fitted pKa for niclosamide of 7.12. B Same data as in $\mathbf{A}$ but axes changed to perhaps a more easily evaluated form. Plotted is the Supernatant Nic concentration [Nic] $(\mu \mathrm{M})(\mathrm{UV} /$ $\mathrm{VIS}$ ) versus supernatant $\mathrm{pH}$ at $20{ }^{\circ} \mathrm{C}$. Again, also included is the $\mathrm{pHp}$ theory for $\mathrm{pK}_{\mathrm{a}}$ of 7.12 and limiting $\mathrm{Nic}_{\mathrm{OH}}$ intrinsic solubility of $2.53 \mu \mathrm{M}$ at $\mathrm{pH}$ 3.66. As shown on the graph, a $20 \mu \mathrm{M}$ prophylactic solution can be made at $\mathrm{pH}$

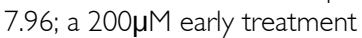
throat spray can be made at $\mathrm{pH} 9.01$, and the concentration can be raised to $300 \mu \mathrm{M}$ at $\mathrm{pH}$ 9.19. The amount of Niclosamide in solution can increase to 703.6 $\mu \mathrm{M}$ at $\mathrm{pH} 9.63$ (Tabulated values are given in Table SI Supplemental Information).

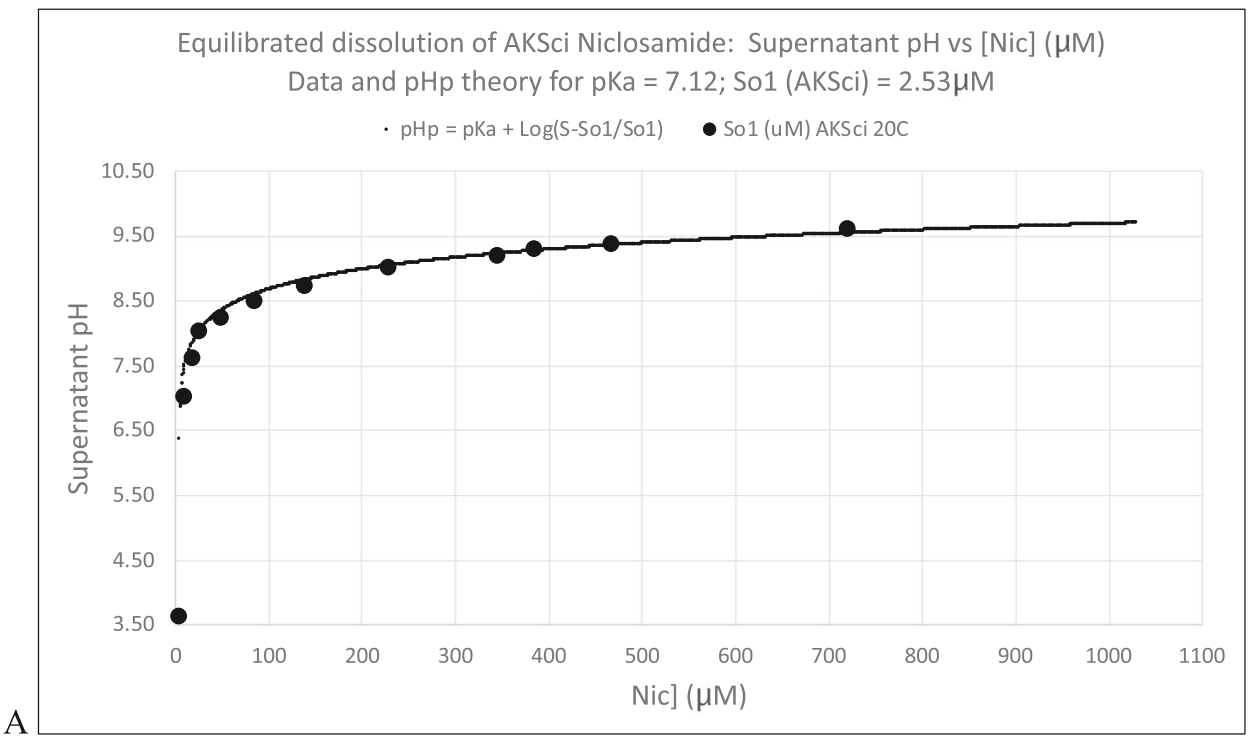

A

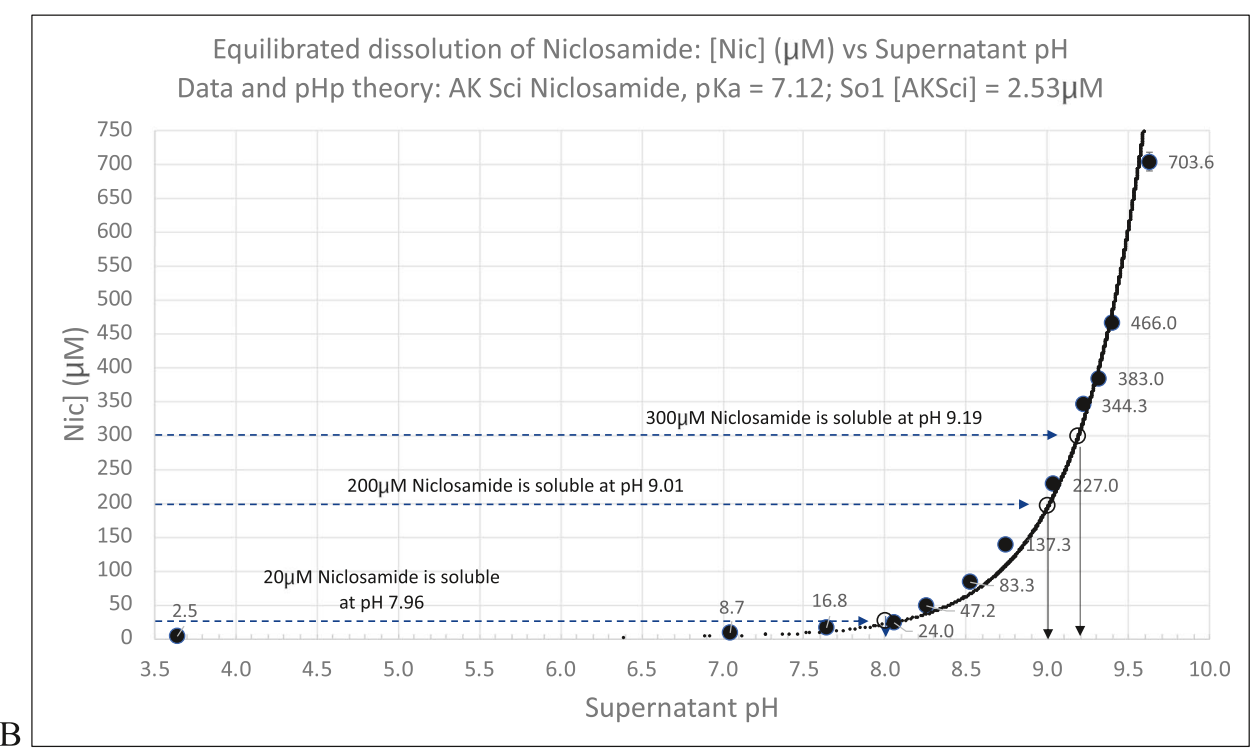

supernatant niclosamide concentration was measured by UV/Vis spectrophotometer.

\section{RESULTS}

\section{Equilibrium Dissolution of Niclosamide versus pH}

Shown in Fig. 2 is a photographic image of $20 \mathrm{~mL}$ scintillation vials containing the equilibrated niclosamide solutions measured by UV/Vis at $333 \mathrm{~nm}$ peak (by Mettler Toledo UV5nano cuvette Spectrophotometer) as equilibrated stirred samples. It is clear, as with the calibration standards (see Supplemental Information S3.2) that the characteristic yellow coloration of the solution gradually increased with increasing $\mathrm{pH}$.
A $300 \mu \mathrm{M}$ sample "standard" made by ethanol solventexchange is shown for comparison (far right). In the final formulation, the preferred method of preparing the niclosamide-in-buffer solutions would be by this ethanol injection and solvent-exchange of an ethanolic niclosamide solution into the buffer. This would allow the ethanolic solution to be pre-filtered to remove the small amount $(<2 \%)$ insoluble material in the AK Sci material.

For convenience, shown in Table SI in Supplemental Information are the nominal and measured $\mathrm{pH}$ and average supernatant Niclosamide concentrations [Nic] $(\mu \mathrm{M})$ corresponding to each of the vials in Fig. 2. Averages and Standard Deviations were taken from five $\mathrm{UV} / \mathrm{Vis}$ measurements at each $\mathrm{pH}$ and the blanks subtracted. Additional re-checks to the original series were taken in the steep part of the curve at pHs 9.1, 9.3 and 
9.5 (data not shown). After addition and equilibration of niclosamide in solution, the $\mathrm{pH}$ values were fairly stable compared to the nominal $\mathrm{pH}$.

The concentrations of these same supernatant solutions are plotted in Fig. 3A for each sample (standard deviations are within the size of the symbols). With increasing measured $\mathrm{pH}$ from 3.66 to 9.63 there is a concomitant increase in the supernatant concentration of niclosamide in solution.

This data is well fitted in form and position by the pHp curve according to this equation (see also Eq. 4 in Supplemental Information S2.3):

$\mathrm{pHp}=\mathrm{pKa}+\log \left(\mathrm{S}-\mathrm{S}_{\mathrm{o}}\right) / \mathrm{S}_{\mathrm{o}}$

where, $\mathrm{S}_{\mathrm{O}}=$ the molar intrinsic solubility of the undissociated acid $\left(\mathrm{Nic}_{\mathrm{OH}}\right)$, and $\mathrm{S}=$ the molar concentration of the salt form in solution $\left(\mathrm{Nic}_{\text {-ve }}\right.$ ), using the measured $\mathrm{S}_{\mathrm{o}}$ intrinsic solubility of niclosamide at $\mathrm{pH} 3.66$ of $2.53 \mu \mathrm{M}$ and a value for the pKa of 7.12. How each curve depends on nominal pKas and intrinsic solubilities is given in Supplemental Information, Fig. S4.

In order to evaluate the data and compare to theory in what is, perhaps, a more easily evaluated form, the same data as in Fig. 3A is replotted in Fig. 3B with the axes switched. As can be seen, the supernatant niclosamide concentration $([\mathrm{Nic}] \mu \mathrm{M})$ increased slowly over the lower $\mathrm{pH}$ range from 3.66 to just above 8 , but then showed the expected more rapid rise in concentration from 8.5 to 9.63 , where some of the highest values measured (and re-checked) for equilibrated samples were $704 \mu \mathrm{M}$. This data and analyses clearly demonstrate the potential for creating simple, more concentrated, solutions of niclosamide for the nasal and throat sprays.

The added horizontal lines in Fig. 3B show that, for prophylactic use, a $20 \mu \mathrm{M}$ solution can be made at $\mathrm{pH} 7.96$, which is within the normal $\mathrm{pH}$ of the nasaopharynx (43). For the early treatment throat spray, and as preclinical animal studies and then human studies proceed to dose escalation, a $200 \mu \mathrm{M}$ solution is readily achieved at $\mathrm{pH}$ 9.01, and the solution concentration of niclosamide can actually be raised to $300 \mu \mathrm{M}$ at only $\mathrm{pH} 9.19$. In the oral cavity a higher $\mathrm{pH}$ is expected to be tolerated and this is where a higher niclosamide concentration is perhaps required for already infected epithelial cells and replicated and secreted lipid-coated virus particles embedded in the mucin secretions.

Comparing the pHp curve to the Henderson Hasselbalch curves (as in Supplemental Information, Figure S3) shows that the data are actually consistent with the prediction of the amount in solution. For example, the amount of the acid in solution is measured to be $2.53 \mu \mathrm{M}$, and so, for a pKa of 7.12, the total amount of niclosamide in solution from the pHp curve should be $2 \times 2.53 \mu \mathrm{M}=5.08 \mu \mathrm{M}$; the $\mathrm{UV} /$ Vis measured value at $\mathrm{pH} 7.05$ is fairly close at $8.68 \mu \mathrm{M}$. Importantly for the nasal and throat spray application is that the amount of niclosamide in solution increases with increasing $\mathrm{pH}$ and was measured to be $\sim 704 \mu \mathrm{M}$ at $\mathrm{pH} 9.63$ where, as shown in Supplemental Information Fig. S3, the dominant species is almost $100 \% \mathrm{Nic}_{\text {-ve }}$. Therefore, these high $\mathrm{pH}$ values are expected to represent the solubility of that charged salt, i.e., 278 times more niclosamide is in solution at $\mathrm{pH} 9.63$ than at $\mathrm{pH} 3.66$, and this is all because of its pKa of 7.12 and a value for $S_{o}$ of $2.53 \mu \mathrm{M}$.

Because we are also interested in the amounts in solution in cell culture, preliminary measurements were also made by incubating the excess AK Sci niclosamide at $37^{\circ} \mathrm{C}$ for pHs. Values were between 1.5 and $2 \times$ higher than reported at $20^{\circ} \mathrm{C}$ in Fig. 3B, namely: $6.06 \mu \mathrm{M}$ at $\mathrm{pH}$ 3.66; $32.0 \mu \mathrm{M}$ at $\mathrm{pH} 7.63 ; 68 \mu \mathrm{M}$ at $\mathrm{pH} 8.26$; and $124.5 \mu \mathrm{M}$ at $\mathrm{pH} 8.57$. These data will be more fully reported in new studies that are currently underway that aim to determine the $\mathrm{CC}_{50}$ for human nasal epithelial cells exposed to niclosamide and if and to what extent niclosamide can in fact inhibit viral replication in human cells for comparison to literature where, so far, there is only data in vero6 and Calu3 cells $(4,35,49)$.

\section{Rates of Dissolution for AK Sci and Sigma Niclosamide Powder}

Having measured the equilibrium values of the amounts of niclosamide that can dissolve over a range of $\mathrm{pH}$, the next series of experiments were to more carefully quantify the rates of dissolution of the AK Sci and Sigma powder that was lightly ground and suspended in buffer solution.

Initial morphology of the AK Sci and Sigma niclosamide powder

First, it is instructive to examine the initial morphologies of the as-supplied AK Sci and Sigma niclosamide powder because it is the inherent morphology and, indeed, the mixed morphologies of the original as-supplied powders that explain their dissolution behaviors and different final equilibrated concentrations when in contact with undissolved excess material. In Fig. 4 are photographic microscope images of as supplied AK Sci and Sigma powder after resuspending in water to disperse, and bath-sonicating to help break up aggregates for better visualization of individual particles.

For the AK Sci material, it appears as a block-like morphology. Characteristic sizes, as length and width of individual particles, range from 1-10 $\mu \mathrm{m}$ with an average 
Fig. 4 Optical microscope photographic images of: A AK Sci niclosamide powder showing a block-like morphology, and B Sigma niclosamide powder showing a mixture of block-like and needle-shaped morphologies. (Bright field, $40 \times$ objective lens, with Köhler illumination). The needle shaped morphology is characteristic of the low solubility monohydrate reported by van Tonder et al. (I).
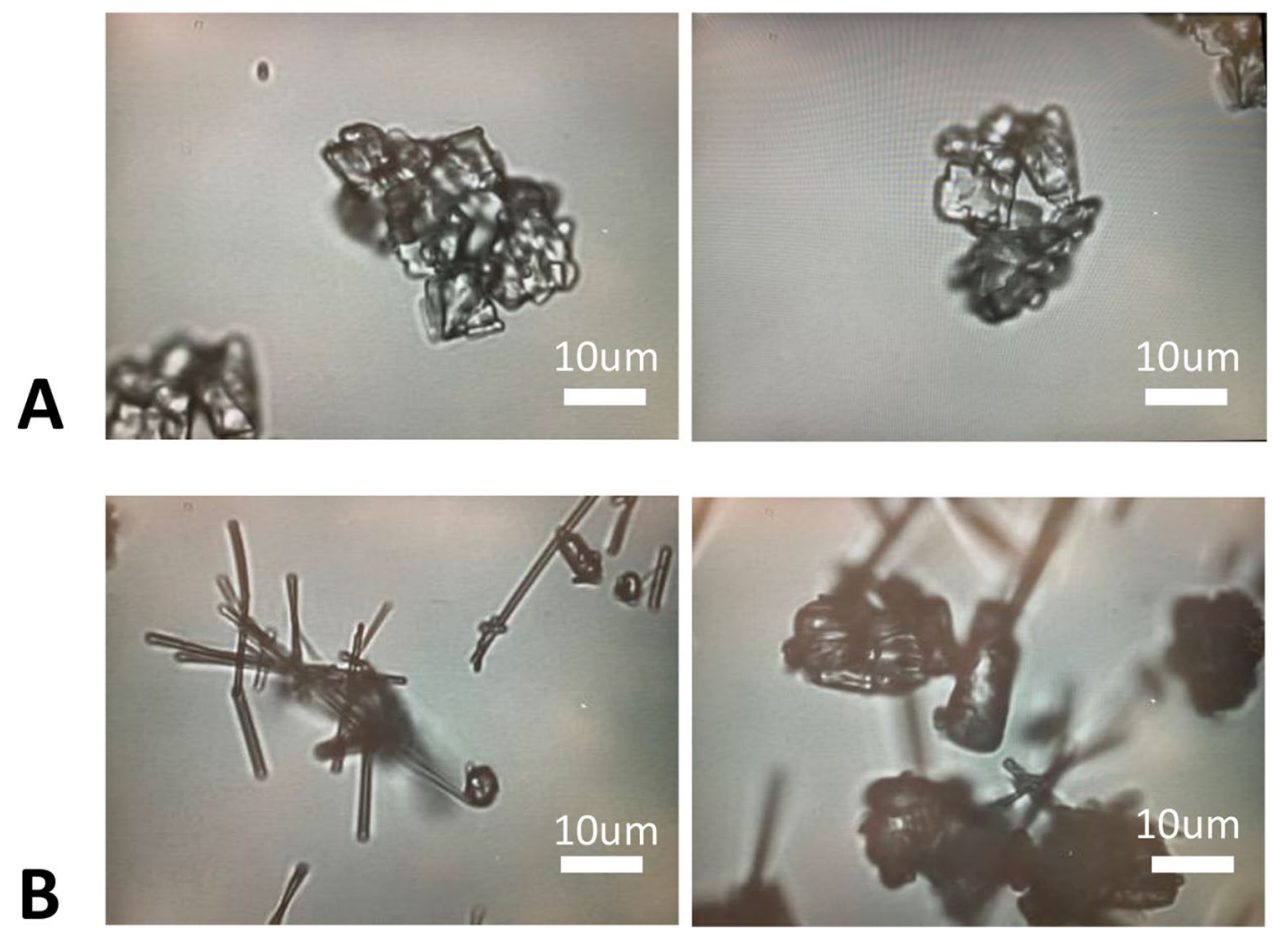

size (from a series of micrographs) of $4.3 \mu \mathrm{m} \pm 2.2 \mu \mathrm{m}$. Interestingly, any needle-shaped crystals that are characteristic of the low solubility monohydrate, as shown by van Tonder $e$ t al. (2) are noticeably, and importantly, absent from this AK Sci supplied material. This is consistent with the AK Sci product being a solid form with a higher intrinsic solubility than the stable monohydrate. In contrast, the as-received Sigma niclosamide showed mixed block-like and needle-shaped morphologies and so already contained some of the assumed low solubility monohydrate polymorph. The extent to which each of these morphologies was maintained for excess material in equilibrium after dissolution across the whole $\mathrm{pH}$ range is shown later for Sigma and AK Sci in Fig. 14A and $\mathrm{B}$ and underlies the difference in final concentrations across the whole $\mathrm{pH}$ range summarized in Fig. 15.

(Note: While efforts were made to ask for details from AK Sci and Sigma, manufacturers were reluctant to divulge any information about product manufacture and post synthesis processing such as precipitation or recrystallization conditions, solvents, and potential solvates. Thus, the nature of the block-like morphology of the powdered material obtained from AK Sci and the mixed morphologies of the Sigma sample remains, as yet unknown).

\section{Dissolution of AKSci and Sigma Niclosamide at the Different pHs and Optimized Stirring}

While a more quantitative analysis would measure the surface area per gram of drug powder, all samples were ground with the pestle and mortar and so were somewhat standardized to a relatively fine powder. Therefore, for the same material the only variables are total mass and mixing. Samples were weighed to within $10 \%$ of $3.27 \mathrm{mg}$, which, in $10 \mathrm{mLs}$ gives a total equivalent $\sim 1 \mathrm{mM}$ niclosamide, and so is in excess of all expected saturated supernatant concentrations. Since the mass added and mixing were similar for each pH sample tested, Fig. 5 shows that the Supernatant Niclosamide Concentration $[\mathrm{Nic}](\mu \mathrm{M})$ versus time (mins) for the lower five dissolution curves gave a fairly quantitative measure of niclosamide dissolution increasing in rate with each $\mathrm{pH}$ of $8.62,8.72,9.06,9.36$, and 9.44 .

As shown earlier in Fig. 3B, below pH 8.5, the equilibrium saturation concentrations are still quite low (although still distinguishable from slight noise of the instrument), and so we focused here on the higher $\mathrm{pH}$ range $8.62-9.5$ for a nominal $1 \mathrm{mM}$ of the AK Sci material. Fitted logarithmic rates for each of these lower dissolution curves (not shown on the graph) were found to increase with increasing $\mathrm{pH}$ as quantified by the pre-ln factor for the logarithmic fits for micromolar versus time in mins. These were, 22.1 (at pH 8.62); 24.3 (at pH 8.78); 34.5 (at pH 9.06); 71.1 (at pH 9.36) and 69.0 (at $\mathrm{pH} 9.44$ ).

During these first experiments for $\mathrm{pH} 8.62-9.5$, the magnetic stirbar was relatively small compared to the diameter of the scintillation vial, (about half) 
Fig. 5 Dissolution curves for the different $\mathrm{pHs}$ using the nanodrop UVIVIS spectrometer (ThermoFisher) over the first I h after addition of dry powdered niclosamide. Data is given for the higher $\mathrm{pH}$ range $8.62-9.5$ for a nominal total I $\mathrm{mM}$ of the AK Sci material. The top two curves, show the (empirical) effect of more optimized stirring, that increases the dissolution rate by $\sim 33 \%$, represented by AK Sci and a second supplier of niclosamide, Sigma. AK Sci niclosamide reached $564 \mu \mathrm{M}$ at $3 \mathrm{~h}$ and Sigma niclosamide reached $680 \mu \mathrm{M}$ at $3 \mathrm{~h}$ at this $\mathrm{pH}$ of 9.5 .

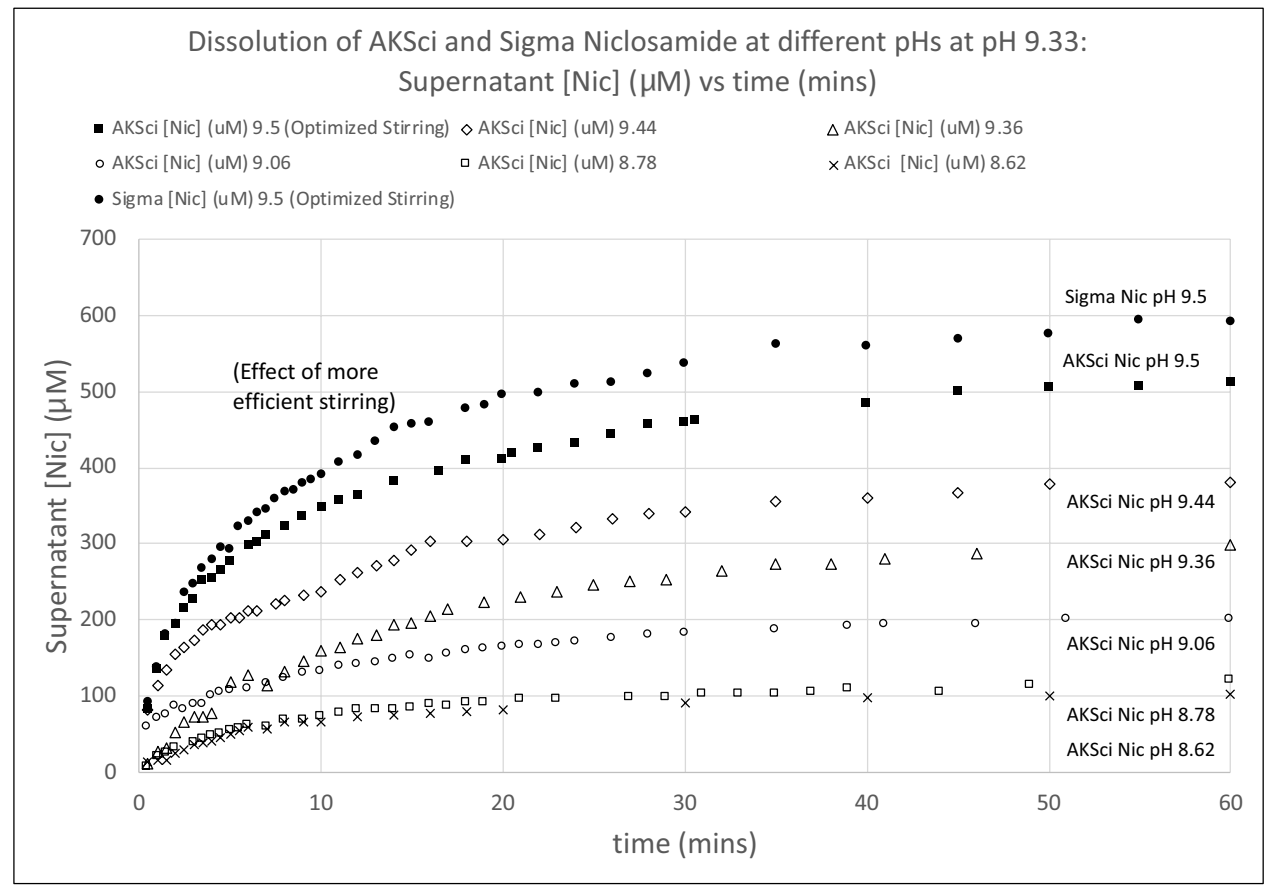

and the rotation speed was medium. In order to, at least empirically, explore the effect of stirring speed, a slightly larger magnetic stir bar was chosen such that the size of the magnetic stirbar was standardized to about $75 \%$ of the diameter of the vial keeping the stirring rate at medium to provide a controlled vortex with no air entrainment. Under these conditions, the dissolution rate of AK Sci niclosamide was increased by about a factor of $37 \%$ i.e., from $\mathbf{6 9 . 0}$ $\ln (\boldsymbol{x})$ (at $\mathrm{pH} 9.44)$ to $94.6 \ln (\boldsymbol{x})$ at the similar $\mathrm{pH} 9.5$ Also shown, the Sigma niclosamide dissolved slightly faster at $113.8 \ln (x)$. For all subsequent dissolution studies, this was the optimized stirring "standardized" set up.

From these data in Fig. 5, the initial dissolution rates were also determined over the first $3 \mathrm{~min}$ as intrinsic concentration units i.e., micromoles of niclosamide dissolved per micrograms of niclosamide in the vial per second $(\mu \mathrm{M} / \mathrm{mg} . \mathrm{s})$. The data is shown in Supplemental Information section S5 in Fig. S9, where initial dissolution rates increased with increasing $\mathrm{pH}$ of the supernatant. The initial rates of niclosamide dissolution are also plotted versus the equilibrium supernatant niclosamide concentration [Nic] $(\mu \mathrm{M})$ in Fig. S10, showing that, as expected from nucleation theory, (e.g., Epstein and Plesset (64-67) they are proportional to the equilibrium solubilities, especially for the higher pHs and hence higher amounts in solution.

\section{Precipitation from Supersaturated Solution and Their "Natural" Morphologies}

\section{Equilibrium Dissolution of AK Sci Niclosamide Precipitated from Supersaturated Solution versus Supernatant $\mathrm{pH}$}

As used to accurately make the control solutions, the second way to achieve a solution of niclosamide is to first dissolve the niclosamide in a water-miscible solvent (like ethanol, or acetone, DMSO, or DMA) and exchange the solvent for the aqueous anti-solvent at a final concentration where the niclosamide is still soluble (see above, "Solvent Exchange Technique" in Methods). This technique can also be used to create normal solutions and also, as in this case, supersaturated solutions from which the niclosamide can (eventually) precipitate and form whatever the stable solid morphology is at that $\mathrm{pH}$. Thus, in these experiments, levels of supersaturation were explored and compared to the parent AK Sci material, including the final equilibrium niclosamide concentrations of the supernatants as a function of supernatant $\mathrm{pH}$ (Fig. 6, open symbols). Thus, niclosamide from the same source (AK Sci) was first dissolved into ethanol at $30 \mathrm{mM}$ and then injected into each buffer at 2-5 times the excess concentration of that measured solubility, allowed to precipitate, and fully equilibrate for 8 days. It was then filtered through a $0.22 \mu \mathrm{m}$ filter prior to taking the clear supernatant for UV-Vis measurement. 
Fig. 6 Supernatant Niclosamide concentrations [Nic] $(\mu \mathrm{M})$ versus supernatant $\mathrm{pH}$ for $\mathrm{AK}$ Sci Niclosamide precipitated from supersaturated solution (open symbols) compared to $\mathrm{pHp}$ theory with $\mathrm{pKa}=7.12$, and intrinsic solubility $\mathrm{S}_{0} 2=1.01 \mu \mathrm{M}$ (dashed line). Also shown is the AK Sci niclosamide dissolved from powder (from Fig. 3B) (filled symbols) for the same pKa and where its intrinsic solubility Sol $=2.53 \mu \mathrm{M}$ (solid line).

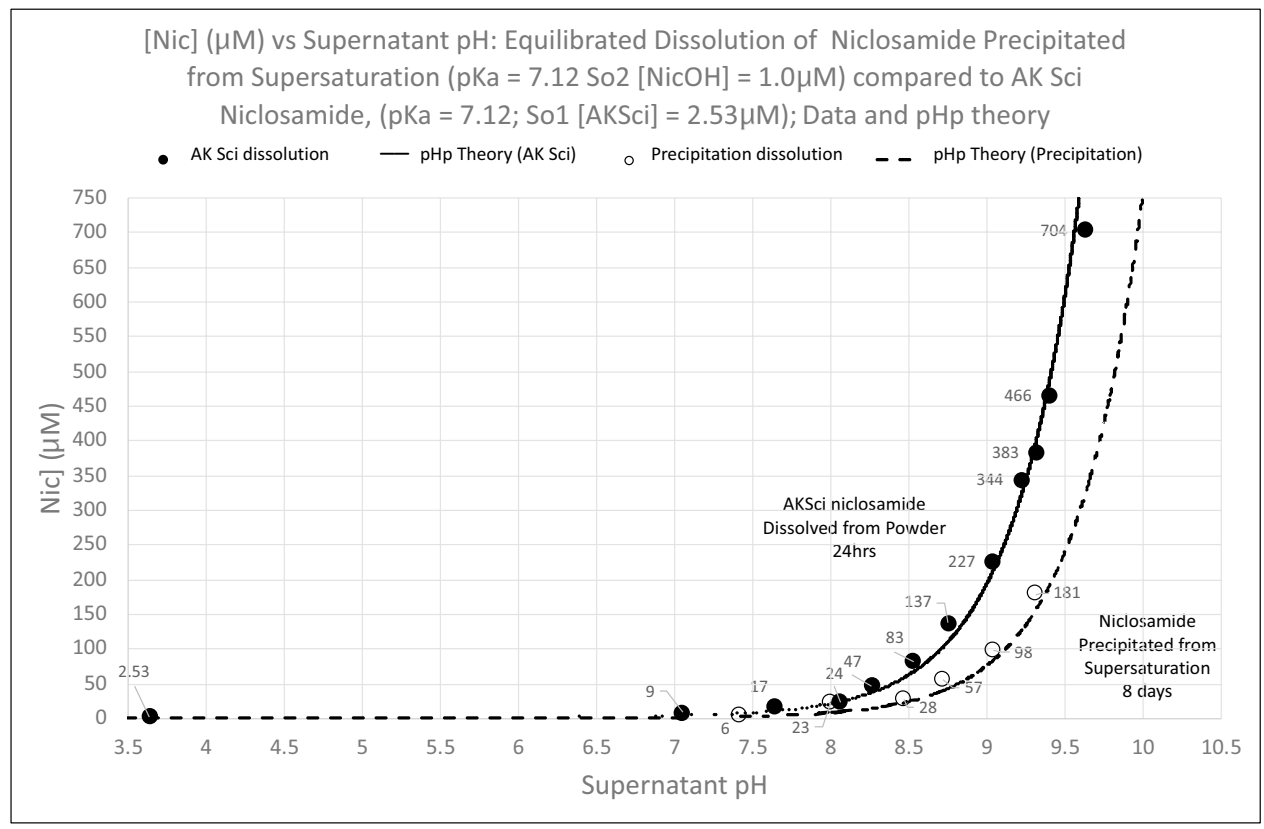

For comparison, also shown in Fig. 6 (filled symbols), is the series of AK Sci niclosamide dissolved from ground-powder, as given earlier in Fig. 3B. As shown in Fig. 6, the precipitated niclosamide has a much lower supernatant niclosamide concentration in solution than the parent compound, that is simply dissolved in each pH buffer.

The solid and dashed lines are the pHp curves for each system where the pKa is the same one (7.12) derived from fitting the AK Sci dissolution data from Figs. 3A and B. The pHp curve for the precipitated niclosamide (dashed curve), uses the measured value of the intrinsic solubility of the fully protonated acid $\mathrm{S}_{\mathrm{o}} 2$ of $1.01 \mu \mathrm{M} \pm 0.26 \mu \mathrm{M}$, measured at $\mathrm{pH} 3.66$. Also shown is the pHp theory for the dissolution of the parent $\mathrm{AK}$ Sci niclosamide (solid curve, from Fig. 3B), using $\mathrm{S}_{\mathrm{O}} 1$ of $2.53 \mu \mathrm{M} \pm 1.0 \mu \mathrm{M}$. Since the $\mathrm{pKa}$ is the equilibrium balance between the acid and salt forms in solution, it is satisfying that the same pKa of 7.12 can be used to fit both sets of data when the measured value $\left(\mathrm{S}_{\mathrm{o}} 2\right.$, of $1.01 \mu \mathrm{M})$ for the intrinsic solubility of the precipitated $\mathrm{Nic}_{\mathrm{OH}}$ is used.

What this data shows is that niclosamide, when precipitated from supersaturated solution, equilibrates to a much lower final supernatant niclosamide concentration, which is presumably reflective of a more stable polymorph at each $\mathrm{pH}$. Prior to precipitation, it was possible to achieve supernatant concentrations (kinetic solubility) as high as $3 \mathrm{mM}$ niclosamide at the highest $\mathrm{pH}$ of 9.5 (reading off the dashed line at $250 \mu \mathrm{M}$ ), -a supersaturation of $\sim 3 \mathrm{mM} / 250 \mu \mathrm{M}=12$ times. The data shows that, while the AK Sci niclosamide polymorph was relatively stable in solution at the high concentrations achieved, precipitation from supersaturated solution presumably forms the water-hydrate at each $\mathrm{pH}$, that still does have a $\mathrm{pH}$ dependence for its now thermodynamic solubility (to be checked in future research (62)).

\section{Supersaturated Solutions form a Series of Niclosamide Morphologies of Reduced Solubility}

Corresponding to the supernatant solubilities given in Fig. 6, samples of the precipitated material were taken and viewed under optical microscopy. Shown in Fig. 7 are the photographic microscopic images of precipitated particles at each supernatant $\mathrm{pH}$ as full screen images using a $40 \times$ bright field objective.

At $\mathrm{pH} 7$, where the low solubility protonated acid makes up almost $50 \%$ of the niclosamide in solution (see Henderson Hasslebalch curve in Supplemental Information, Fig. S3), niclosamide precipitates as flat particulate sheets formed in the stirred aqueous buffer that make a mass of gel-like particles in suspension. Macroscopically, these are the characteristic white particles seen swirling in the vial (in Supplemental Information Fig. S1). This flat sheet, particulate, gel-like morphology persists at $\mathrm{pH}$ 7.5, 8.0, 8.25 and 8.5. Being formed from a 1:99 dilution of ethanolic niclosamide that is exchanged for the excess $\mathrm{pH}$ buffer under rapid stirring, some sheets are observed to fold, as in the image for $\mathrm{pH}$ 8.25. Then, at $\mathrm{pH} 8.75$, the morphology makes a transition to the more usual monohydrate needle-like polymorph that is characteristic of the precipitated particles at pH 9.0 and 9.3, where the deprotonated niclosamide salt is the dominant species. 


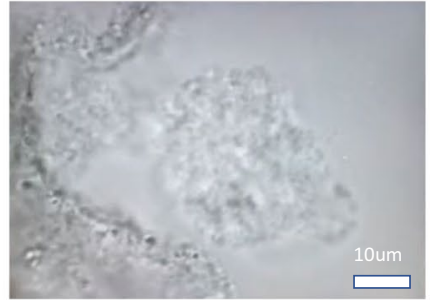

pH 7.0

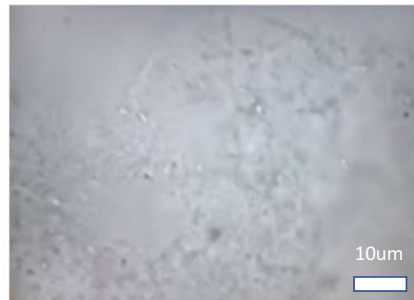

pH 8.5

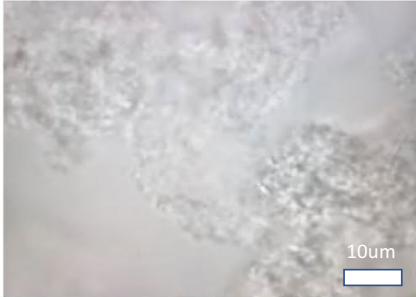

$\mathrm{pH} 7.5$

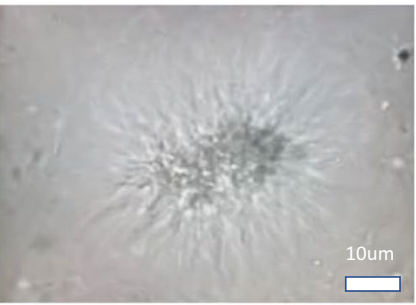

$\mathrm{pH} 8.75$

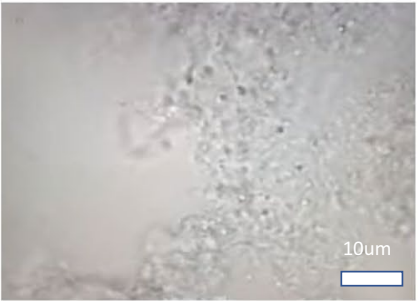

$\mathrm{pH} 8.0$

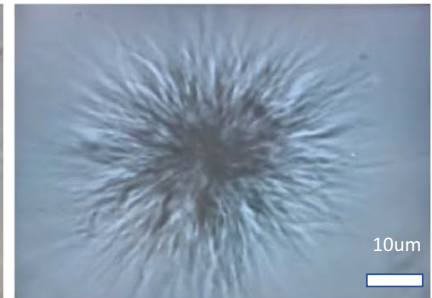

pH 9.0

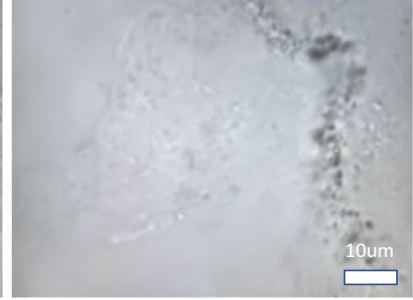

$\mathrm{pH} 8.25$

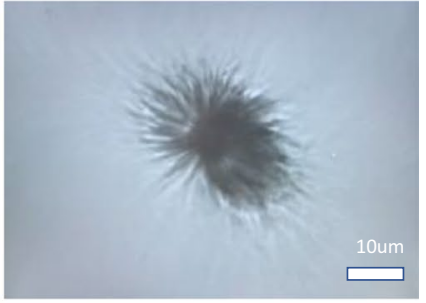

$\mathrm{pH} 9.3$

Fig. 7 When precipitated from supersaturated solutions AK Sci niclosamide forms a series of particle morphologies of reduced solubility such that the amount in a solution that changes with pH. Shown are optical microscope images (bright field, $40 \times$ objective lens, with Köhler illumination) taken from the precipitated samples at each indicated $\mathrm{pH}$. Scale bar is $10 \mu \mathrm{m}$.

What these microscopic images demonstrate then, seemingly for the first time, is that these most stable niclosamide hydrates (2) not only have a $\mathrm{pH}$ dependence to the amount of niclosamide in solution but also have a $\mathrm{pH}$ dependent morphology.

\section{Niclosamide Gel-Like Particles Also Display a Strong Hydrophobicity and Coat Gas Bubbles}

In some images of the samples of the precipitates, dark structures were often observed. This is a new and interesting, but not unexpected observation that the niclosamide gel-like particles also displayed a strong hydrophobicity. As shown in Fig. 8, what these represent are gas microbubbles that follow the crumpled contours of the niclosamide precipitated sheet formed at pHs 7.0, 7.5, 8.0 and 8.5 as the precipitate adheres to the bubble surface. Because the particles are formed by precipitation in a rapidly stirred buffer environment, it seems that gas microbubbles can get trapped. These characteristically optically black masses of air show a complete lack of surface tension (or tension in the surface). That is, rather than being round and exhibiting their usual air-water surface tension and concomitant Laplace pressure, they are deformed to the shape of the niclosamide material. Clearly, as one would expect from niclosamide's low solubility and moderate $\log \mathrm{P}$ of 4 at these $\mathrm{pHs}$, the niclosamide particles are quite hydrophobic (as is air). This was also borne out by the way the particles of powder, when added to a vial for the dissolution test, would rapidly float at the air solution interface unless well shaken to wet the powdered particles and immerse them in buffer suspension.

This kind of zero tension and zero Laplace pressure has actually been observed and well- characterized before for lipid coated gas microbubbles (as solid lipid monolayers) in our micropipette experiments that measured interfacial tensions as well as gas bubble dissolution in undersaturated solutions $(64,68,69)$. Here though, the hydrophobic precipitated niclosamide sheets form a kind of "Pickering gas-emulsion" that is stabilized by the sheet-like particles adsorbed onto the interface between the aqueous and gas phases.

\section{Dissolution of Niclosamide as a Water-Precipitate, and as Recrystallized Material From Acetone and Ethanol}

\section{Dissolution Profiles}

Given the high solubilities observed by the AK Sci material, while other sources and precipitated material readily converted to the less soluble and more stable hydrates, preliminary studies were also conducted to create different cosolvates in order to evaluate their dissolution and crystal morphologies. These studies were conducted to initially compare the samples with the AK Sci and Sigma materials and try to ascertain if they were a water, ethanol or acetone solvate; they did not appear to be so. 
Fig. 8 The niclosamide gel-like particles also display a strong hydrophobicity. Gas bubbles, that were adsorbed or trapped in the microparticles due to stirring during initial mixing and subsequent stirred equilibration, appeared to follow the crumpled contours of the niclosamide precipitated sheet formed at $\mathrm{pHs}$ 7.0, 7.5, 8.0 and 8.5. The precipitate adhered to the bubble surface showing that the interfacial tension and Laplace pressure were zero. (Bright field, $40 \times$ objective lens, with Köhler illumination).
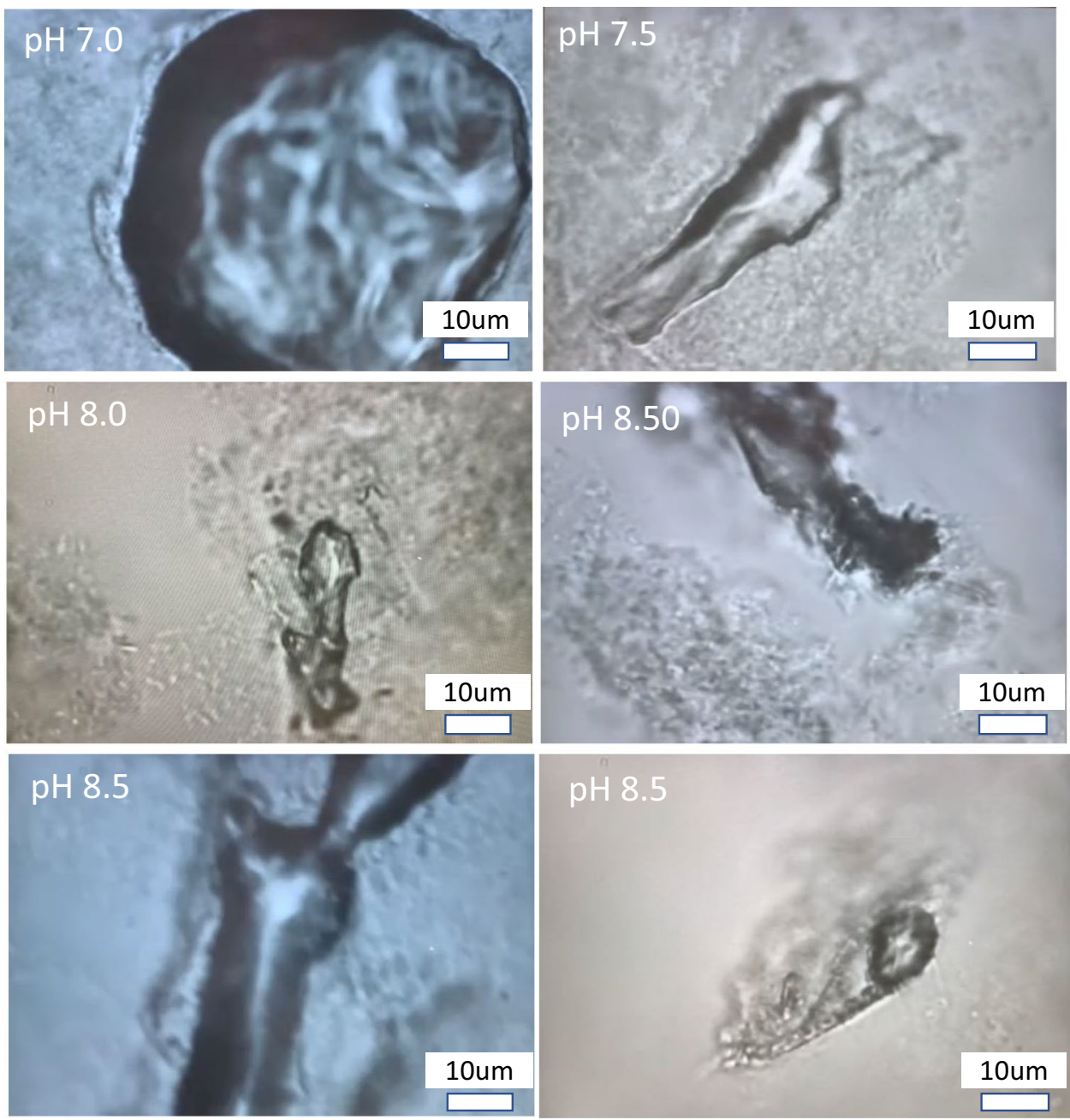

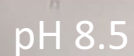

Samples of niclosamide were made from the AK Sci original material as a water-precipitate and recrystallized from Acetone and Ethanol, as described in methods, broadly following those by van Tonder et al. for their well-characterized cosolvates $(2,59,61)$. For dissolution, the same mass of ground material, $3.5 \mathrm{mgs}$, was added to each $10 \mathrm{mls}$ of $\mathrm{pH} 9.3$ buffer in a $20 \mathrm{~mL}$ scintillation vial and stirred with magnetic stir bar. $2 \mu \mathrm{L}$ samples were taken at time intervals and their absorbance measured on a UV/VIS nanodrop spectrophotometer at $333 \mathrm{~nm}$. The concentrations of niclosamide $(\mu \mathrm{M})$ in the supernatant versus time (mins) are plotted in Fig. 9 for the water precipitate, and the recrystallized niclosamide from acetone and ethanol.

Dissolution profiles were therefore determined for each of the dried powdered samples (water precipitated, and acetone and ethanol recrystallized) as well as the equilibrium supernatant concentrations two days after dissolution, all determined at $\mathrm{pH}$ 9.3. As can be seen, the dissolution rates go as $\mathrm{Nic}_{\mathrm{H} 20 \mathrm{ppt}}>\mathrm{Nic}_{\text {Acetone recrys }}>\mathrm{Nic}_{\mathrm{Ethanol} \mathrm{recrys}}$, although the initial rates measured as the intrinsic parameter $\mu \mathrm{M} / \mathrm{mg}$ of material were: $\mathrm{Nic}_{\mathrm{H} 20 \mathrm{ppt}}(0.104$ $\mu \mathrm{M} / \mathrm{mg})>\mathrm{Nic}_{\text {Ethanol-recrys }},(0.0439 \mu \mathrm{M} / \mathrm{mg}) \sim \mathrm{Nic}_{\text {Acetone-recrys }}$ $(0.0369 \mu \mathrm{M} / \mathrm{mg})$.

This data shows that the commercial niclosamide products were likely not recrystallized from acetone (as we were told) because the as-received niclosamide samples attained a much higher concentration in solution $(\sim 550 \mu \mathrm{M}$ for AK Sci) at $\mathrm{pH} 9.3$ than the acetone and ethanol recrystallized material $(79.4 \mu \mathrm{M}, 78.9 \mu \mathrm{M})$. The graph also shows how the water "solvate" that was dried and then re dissolved at $\mathrm{pH} 9.3$ has a similar $(132.6 \mu \mathrm{M})$ solubility to that precipitated in situ as in Fig. 6 of $180.9 \mu \mathrm{M}$. that was slightly higher than the acetone cosolvate $79.4 \mu \mathrm{M}$ and ethanol cosolvate $78.9 \mu \mathrm{M}$.

\section{Crystal Morphologies}

Images of the morphologies of the water-precipitated and acetone- and ethanol- recrystallized samples are shown in Fig. 10. 
Fig. 9 Dissolution of Niclosamide $\left(\mathrm{H}_{2} \mathrm{O}\right.$ precipitate and recrystallized from Acetone and Ethanol) Supernatant Niclosamide concentration [Nic] $(\mu \mathrm{M})$ versus time (min). Also shown are the overall rates and final equilibrium supernatant concentrations of niclosamide several days after dissolution was started.

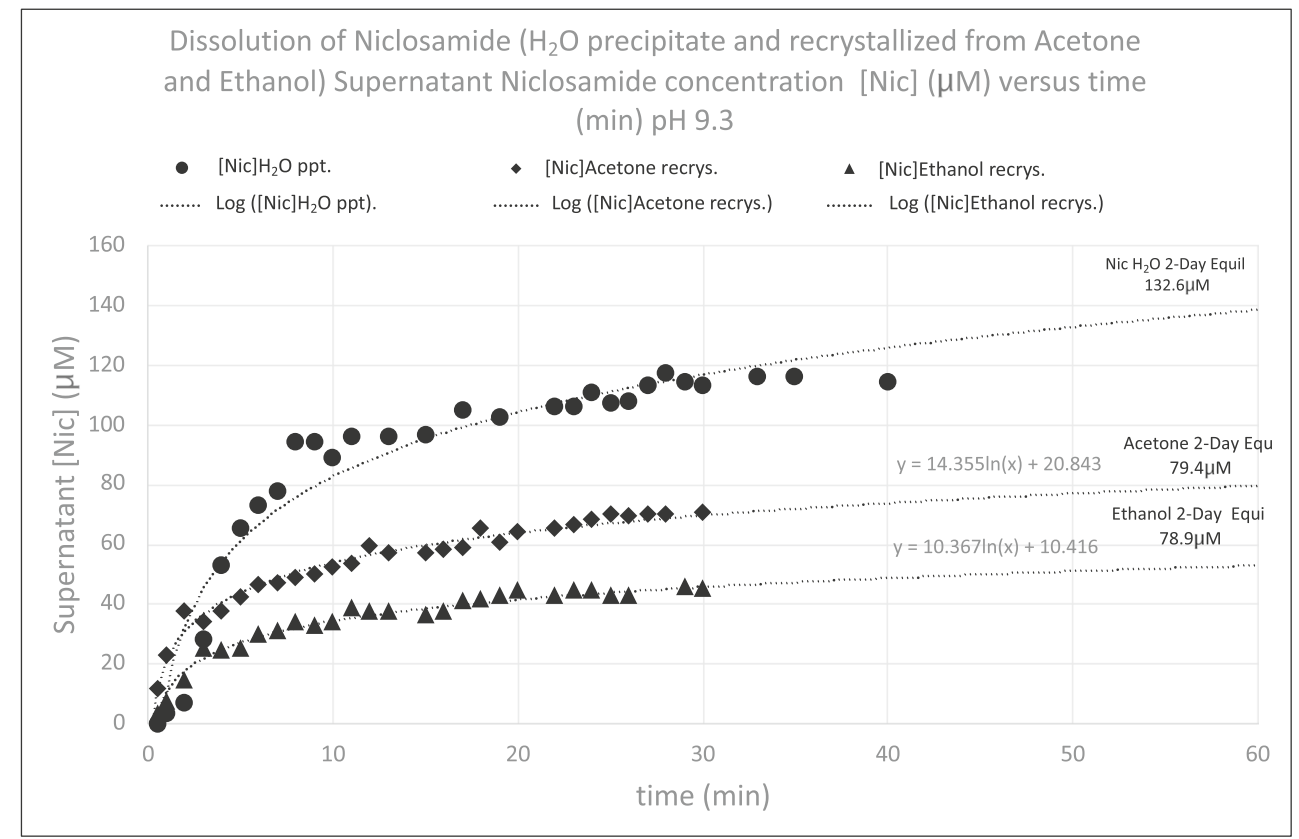

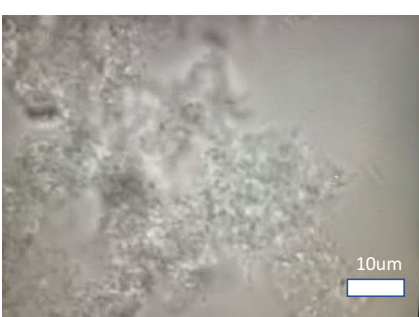

Niclosamide Precipitated from Ethanol into excess pH 7 buffer

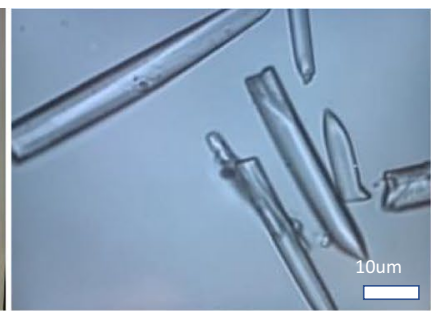

Niclosamide Recrystallized from Ethanol

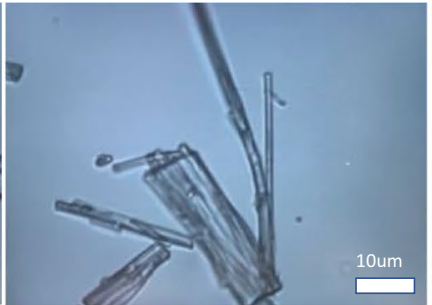

Niclosamide Recrystallized from Acetone

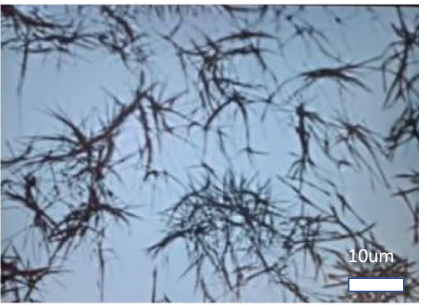

Niclosamide Recrystallized from Dimethyl Acetamide

Fig. 10 Optical microscope images of Niclosamide precipitated from supersaturation, and Niclosamide recrystallized from ethanol, acetone and also dimethyl acetamide. (Bright field, $40 \times$ objective lens, with Köhler illumination).

As shown earlier (Fig. 7), and included again here for comparison, niclosamide precipitated from supersaturated solution (after solvent exchange from an ethanolic solution into excess water at $1 \%$ ethanol) has a very different morphology to the rod-like samples recrystallized from acetone and ethanol. These latter structures are more reminiscent of the methanol and other cosolvates made and characterized by van Tonder et al. (2, 59-61). Additionally, although not measured in dissolution studies, is an image of a sample of niclosamide recrystallized from dimethyl acetamide that had an even finer and more fibrous morphology.

These images taken of the various precipitated and solvent-recrystallized samples further illustrate that the source of niclosamide, including its synthesis and post processing solvent recrystallization or precipitation recovery, can dramatically influence the final solubility of the drug compound and its amount in aqueous solution.

\section{Sigma Niclosamide Dissolution and Overnight Stirring Also Produces Lower Solubility Material with Different Morphology}

\section{Supernatant Images}

As shown above (Fig. 5), the Sigma Niclosamide product readily dissolved and, over a period of $3 \mathrm{~h}$ at $\mathrm{pH}$ 9.5, achieved a niclosamide supernatant concentration of $680 \mu \mathrm{M}$. While slightly more soluble than the AK Sci product (AK Sci was $564 \mathrm{mM}$ ) this is very much in line with the AK Sci material at this $\mathrm{pH}$. However, while the AK Sci material appeared to be generally stable in the presence of excess powdered particles that were in equilibrium with their supernatant solution, as shown in Fig. 11, the Sigma material converted to a much lower solubility solid form when stirring was continued over a period of the next several hours. 
Fig. I I Comparison between (top) Sigma niclosamide freshly dissolved at 3hrs, and (bottom) after 24hrs stirring, showing the clear supernatant converted into a cloudy suspension. The supernatant niclosamide concentration was also decreased, as shown below in Fig. 12.

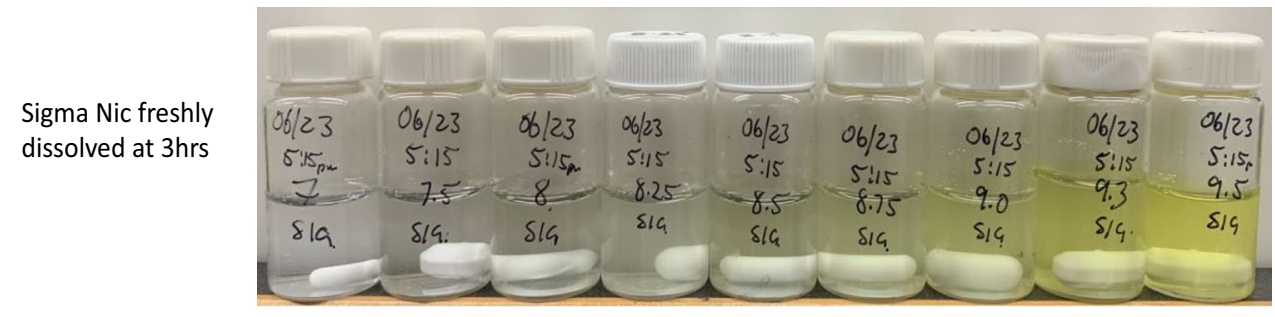

Sigma Nic after 24hrs stirring
Fig. 12 Dissolution of Sigma Niclosamide in $\mathrm{pH}$ buffer at $3 \mathrm{hrs}$ (open diamond symbol in parentheses at $\mathrm{pH} 9.5$ and $68 \mid \mu \mathrm{M}$ ) and 24hrs (filled triangles). Shown is Supernatant Niclosamide [Nic] $(\mu \mathrm{M})$ vs Supernatant $\mathrm{pH}$ and the $\mathrm{pHp}$ curves (dashed curve, fitted with $\mathrm{pKa}=7.12$ and So2 $=1.01 \mu \mathrm{M}$ ). The Sigma data is compared to the AKSci data (filled circles, and solid pHp curve) for the same overnight equilibration. Also shown are photographic images of the same Sigma niclosamide sample in the $20 \mathrm{~mL}$ scintillation vial of the clear supernatant after 3 hr dissolution (clear supernatant at $68 \mid \mu \mathrm{M})$ and then after overnight stirring (cloudy supernatant at $247 \mu \mathrm{M})$.

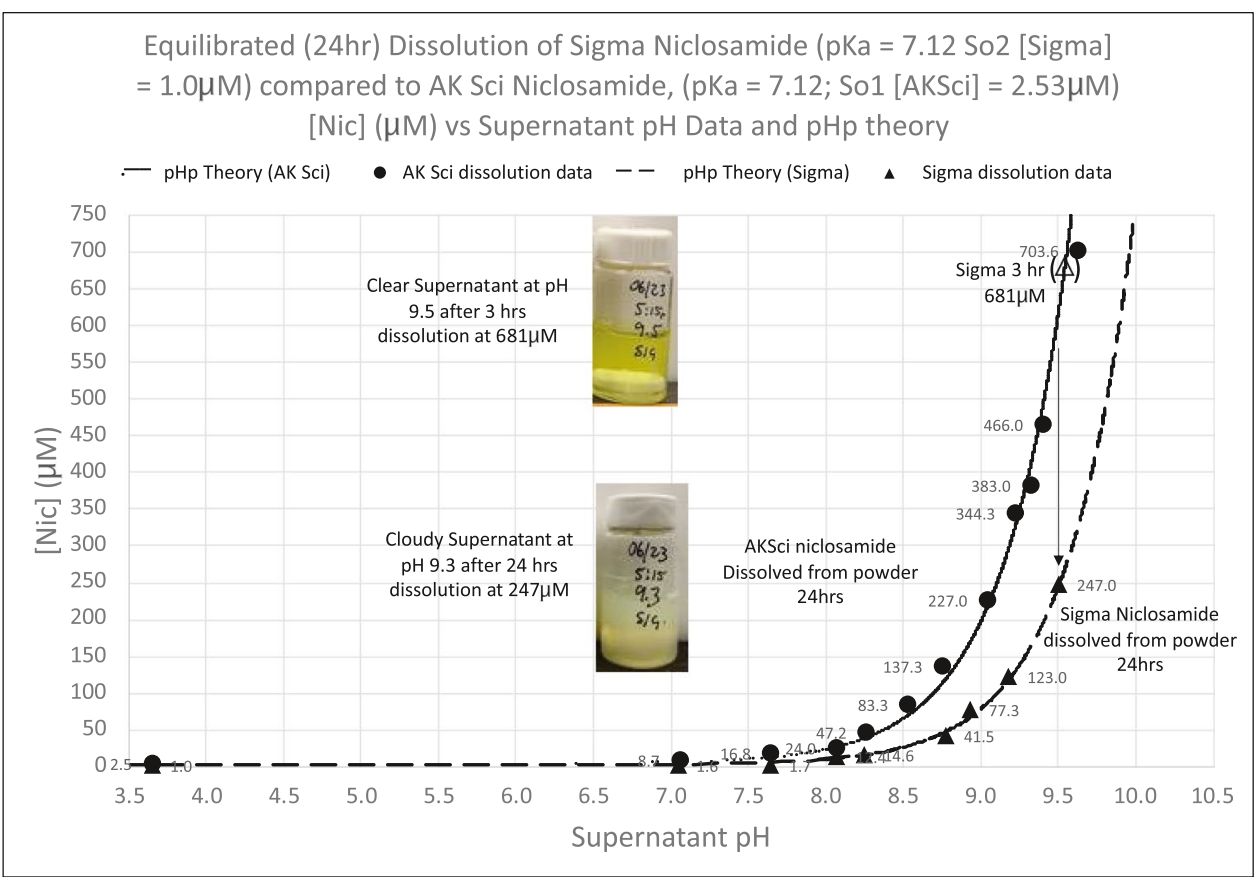

$24 \mathrm{~h}$ after initially starting the dissolution experiment, as shown in Fig. 11, the supernatant went from a clear solution (top image at $3 \mathrm{~h}$ ) to a cloudy suspension (lower image at $24 \mathrm{~h}$ ).

\section{Dissolution Profiles}

When the supernatants were filtered through $0.22 \mu \mathrm{m}$ filters and the supernatant niclosamide concentration was measured by UV/Vis spectroscopy, the supernatant niclosamide concentration had fallen to $198 \mu \mathrm{M}$ at $\sim \mathrm{pH} 9.6,-\mathrm{a}$ loss of material concentration of $482 \mu \mathrm{M}$. Thus, as shown in Fig. 12, when dissolved and equilibrated over the range of pHs 7 to 9.5, the final supernatant concentrations were in a similar range to those measured for niclosamide precipitated from supersaturated solution (in Fig. 6). i.e., they both showed amounts in solution (Sigma Nic at $\mathrm{pH} 9.3=103 \mu \mathrm{M}$; precipitated material $=180.9 \mu \mathrm{M}$ ) consistent with forming the more stable hydrated material. Again, comparing the experimental data to the $\mathrm{pHp}$ model using the same derived pKa of 7.12 to get the initial fit, the measured value of So2 of $1.01 \mu \mathrm{M}$ (dashed line) is used to fit the data that is again consistently lower than the AK Sci polymorph. Comparison is also made with the AK Sci polymorph as both measured data of niclosamide in supernatant solution in equilibrium with excess undissolved material and the pHp curve (solid line) for its intrinsic solubility of $2.53 \mu \mathrm{M}$ and the same pKa of 7.12. 
Fig. I3 Optical microscope images of (left) the Sigma Niclosamide as received from the supplier and (right) after overnight equilibration at $\mathrm{pH} 9.3$ Overnight equilibration of Sigma Niclosamide (right) produced long rod-like and spiky-bundled structures and a background of smaller fainter rods that have a much lower $(\sim 3.5 x)$ solubility than the parent Niclosamide powder.
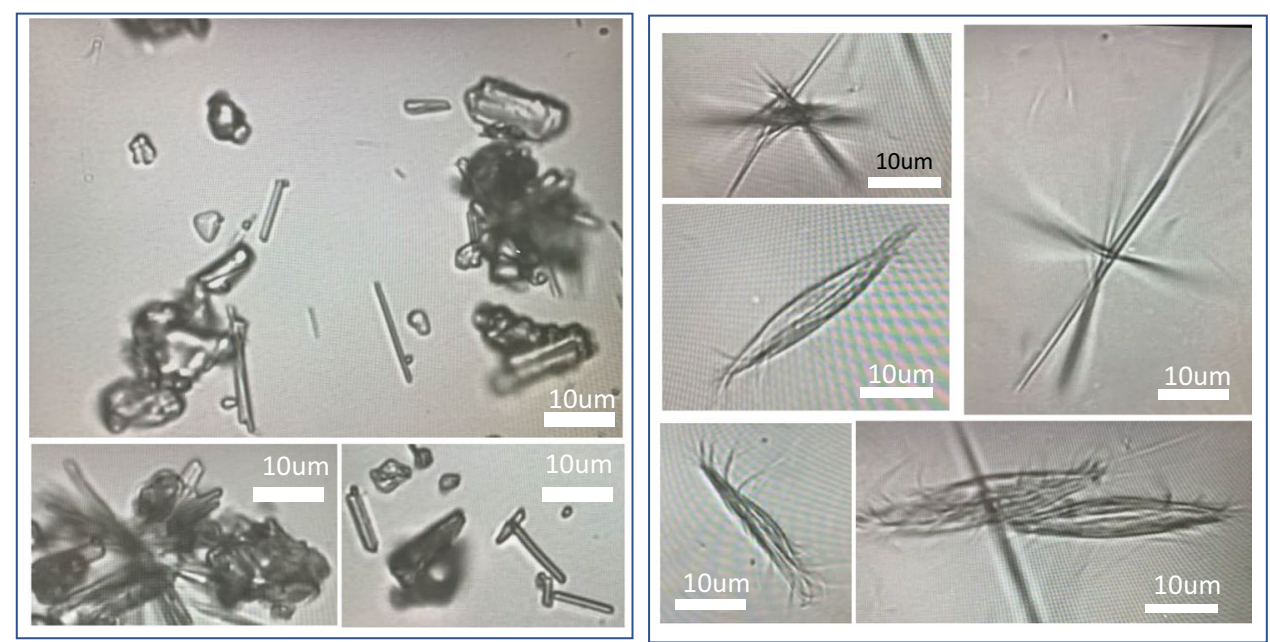

\section{Crystal Morphologies}

Finally, regarding the crystal morphologies for the "Sigma polymorph" as shown in Fig. 13, this overnight equilibration and resulting decrease in supernatant concentration produced a change in particle morphology. The block-and-rod-like particles of the original Sigma niclosamide material (left) were converted to long, more fibrous, and spiky bundled structures (right) with a background of smaller fainter fibers that have a much lower $(\sim 3.5 \mathrm{x})$ solubility than the parent Sigma niclosamide powder.

To investigate this further and compare with the AKSci material, additional optical microscope images were made of excess niclosamide powder equilibrated in buffers across the whole $\mathrm{pH}$ range. These are shown in Fig. $14 \mathrm{~A}$ and B. They clearly explain the difference in dissolution and equilibration behavior between the two supplied Niclosamides. That is, the difference between the Sigma niclosamide product and the AK Sci product is that, while both have an appearance of block-like crystals, the "Sigma polymorph" (Fig. 14A) has a mixture of block-like and needle-shaped morphologies indicating that it already contains a mixture of polymorphic niclosamide including the low solubility monohydrate. This mixture of polymorphs was likely processed by the manufacturer after synthesis by recrystallization or precipitation in different media. Thus, initial dissolution of the excess material is likely to be the block-like, higher solubility, polymorph and then the remaining excess needle-shaped niclosamide initiates the polymorphic recrystallization to the lower solubility monohydrate. Thus, the powdered "Sigma (mixed) polymorph" is more prone to conversion to the more stable monohydrate after overnight equilibration in the pH 9.3 buffer than the "AK Sci polymorph" (Fig. 14B) that only contains block-like morphology and a complete absence of the monohydrate morphology.

As such, the AK Sci niclosamide did not readily convert to the less soluble (needle-shaped morphology) hydrates when incubated for days in the presence of its excess undissolved original powder.

All of the above niclosamide particles (AK Sci, Sigma as supplied, precipitated, converted and recrystallized) would now require crystallographic analyses for measuring polymorphic, hydrate, and amorphous states as could be done by x-ray crystallography and/or FT-IR/ Raman, as well as calorimetric evaluation, as used here for two polymorphic forms of sulindac with very different intrinsic solubilities and therefore different amounts in solution across the whole $\mathrm{pH}$ range (70).

\section{DISCUSSION}

The main results of this study are that (1) Niclosamide has a $\mathrm{pH}$-dependent solubility and dissolution rate, and (2) the intrinsic solubility and the amount of niclosamide in supernatant solution is determined by the nature of the solid form in contact with the supernatant solution and ultimately the most thermodynamically stable crystalline forms. For the "AK Sci polymorph", the intrinsic solubilities of the protonated acid $\left(\mathrm{Nic}_{\mathrm{OH}}\right)$ and unprotonated salt $\left(\mathrm{Nic}_{-\mathrm{ve}}\right)$ are measured to be $2.53 \mu \mathrm{M}$ (at $\mathrm{pH} 3.66$ ) and, at least $704 \mu \mathrm{M}$ at $\mathrm{pH} 9.63$, respectively. Between these two pHs the important quantity is the amount of niclosamide actually in solution. This is therefore determined by the $2.53 \mu \mathrm{M}$ of the acid (at its intrinsic solubility limit) plus the amount of the unprotonated salt that increases with increasing $\mathrm{pH}$. Regardless of polymorph, the amount of niclosamide in aqueous solution follows the predicted $\mathrm{pH}$ dependence 
pH 3.61

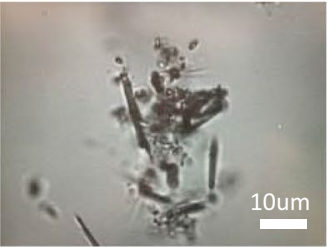

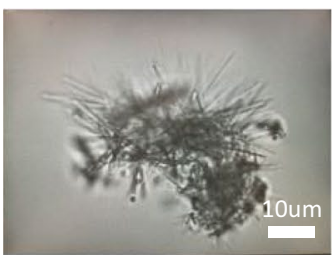

pH 8.56

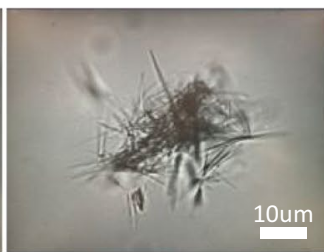

$\mathrm{pH} 7.04$

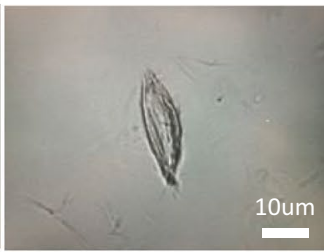

pH 8.77

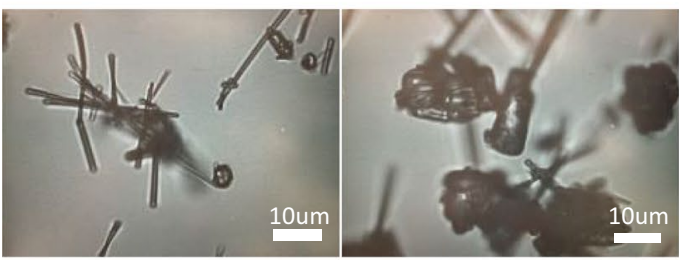

A

Sigma niclosamide: dry powder

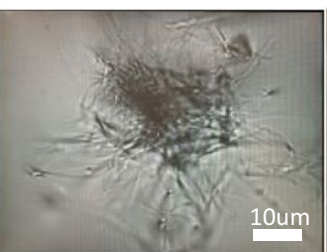

$\mathrm{pH} 7.64$

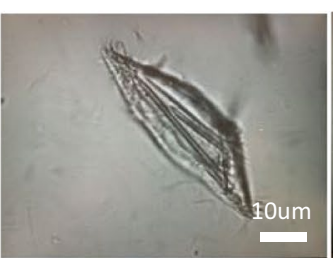

pH 8.93

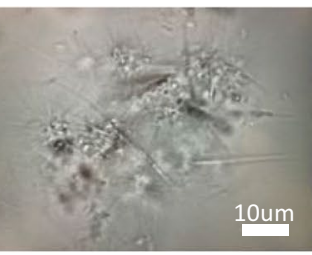

$\mathrm{pH} 8.07$

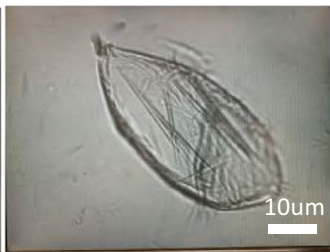

pH 9.18

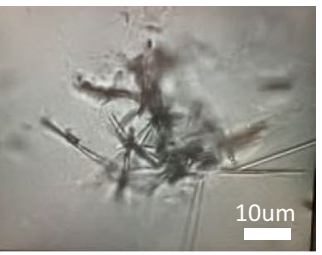

pH 8.25

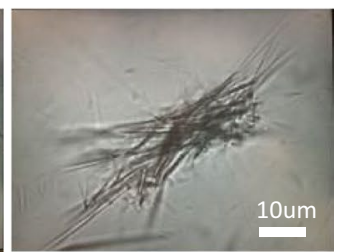

pH 9.51

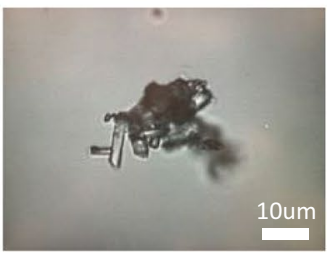

pH 3.65

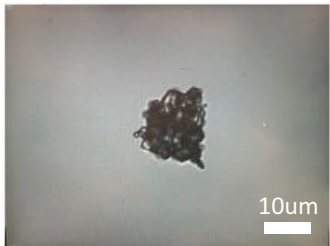

$\mathrm{pH} 8.53$

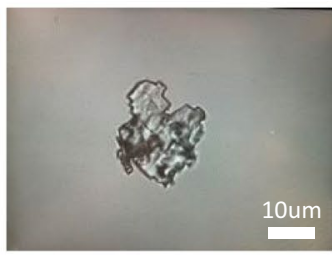

pH 9.40

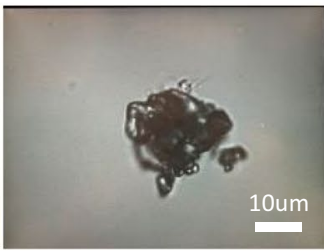

$\mathrm{pH} 7.05$

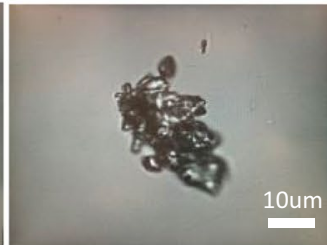

$\mathrm{pH} 8.75$

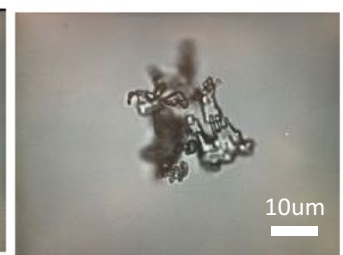

pH 9.63

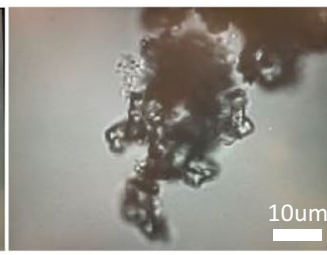

$\mathrm{pH} 7.50$

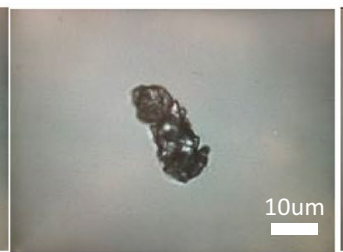

pH 9.04

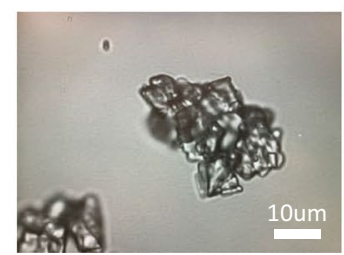

AKSci niclosamide: dry powder

B

Fig. I4 A Optical microscope images of excess Sigma Niclosamide powder particles after overnight equilibration over the whole $\mathrm{pH}$ range from $3.6 \mathrm{I}$ to $9.5 \mathrm{I}$. This overnight equilibration produced long rod-like, needle-shaped, and spiky-bundled structures at all pHs tested showing that the Sigma niclosamide converted to the lower solubility polymorph as the lower solubility monohydrate. These hydrated samples are compared to the dry, as-received, powder from the Sigma supplier that already showed a considerable amount of rod like and needle-shaped crystal morphology in addition to a more block-like morphology. B. Optical microscope images of excess $\boldsymbol{A} \boldsymbol{K} \boldsymbol{S} \boldsymbol{c i}$ Niclosamide powder particles after overnight equilibration over the whole $\mathrm{pH}$ range from 3.65 to 9.63. In contrast to the Sigma niclosamide samples, this AKSci material retained its original block-like morphology at all pHs tested. There was a distinct absence of the prototypical spiky bundled morphology characteristic of the monohydrate. Thus, the AKSci material did not convert to the lower solubility needle-shaped monohydrate. These hydrated samples are also compared to the dry, asreceived, powder from the AKSci supplier that have the block-like morphology. 
from the Henderson Hasselbalch and $\mathrm{pHp}$ models for a given pKa of 7.12 and measured intrinsic solubilities for the AK Sci polymorph of $2.53 \mu \mathrm{M}$ (So1) and that of the low solubility monohydrate polymorph (Sigma, precipitated niclosamide and recrystallized material) of $1.10 \mu \mathrm{M}$ (So2).

Thus, niclosamide is able to take on different polymorphic forms as already established to some extent by van Tonder et al. (2, 59-61), but now showing this for niclosamide from different suppliers, and over a range of pHs. As is well known in pharmaceutics, although identical in chemical composition, polymorphs differ in bioavailability, solubility, dissolution rate, chemical and physical stability and many other properties (70). As encouraged by Llinàs et al. (70), "Despite significant investment in processes to find all the possible polymorphs of active pharmaceutical ingredients (APIs), new polymorphs can suddenly appear without warning. Polymorphs tend to convert spontaneously from less stable to more stable forms, and, therefore, it is best to discover and characterize the stable form as early as possible".

Why this is important is that the low solubility of, especially the monohydrate polymorph, is expected to influence the pharmaceutical performance of the drug product, in particular its solubility limit and dissolution, both of which affect its ultimate local bioavailability. This is especially important in our (and other's (3, 38, $71,72)$ ) applications as nasal and throat sprays. As mentioned above, the most effective way for niclosamide to permeate through the mucin layer that normally covers and protects the underlying nasal and buccal epithelial cells is as a soluble molecule. Also, any aqueous suspension of microparticle material is expected to undergo a conversion to the more stable and least soluble monohydrate form. However, if the solid is filtered out or removed by centrifugation and supernatant decanted off, the remaining supernatant solution retains its high soluble form, unless heterogeneous nucleation triggers the monohydrate. Furthermore, if it is made by solvent exchange, the higher amount in solution is retained because there is no solid material with which it could equilibrate.

Thus, any new formulations of niclosamide that are sought for testing in cells, animals, and especially in humans, and that are expected to deliver molecular niclosamide to target cells, need to take into account the ultimate solubility of any more stable, in this case, monohydrate forms (2). If they occur, or are allowed to occur, they will determine the amount of bioavailable niclosamide that can permeate the mucin in a nasal spray or inhaled administration. In our niclosamide solution approach, the solutions are made by solvent exchange and so there is no particulate material present and so it cannot exert any deleterious equilibrating effects, i.e., the high niclosamide solution concentration is preserved. Given the poor solubility of niclosamide at the neutral and lower $\mathrm{pHs}$ expected in the nasal pharynx, understanding the $\mathrm{pH}$ dependence of niclosamide in aqueous buffers allows a more optimized simple solution to be used for nasal and throat sprays.

Discussed briefly next are the main experimental results associated with the equilibrium and kinetic dissolution experiments for niclosamide into $\mathrm{pH}$ buffers from commercial sources and how this enables making the niclosamide-based nasal and throat sprays. This is followed by brief discussion of the additional scientific findings that when niclosamide is precipitated by solvent exchange or when supernatant solutions (such as those made by the Sigma polymorph) are left in contact with the excess solid material, niclosamide readily reverts to more stable and lower solubility polymorphs. Again, filtering out the excess niclosamide preserves the achieved amount in solution.

\section{Niclosamide solutions can readily be obtained by dissolving niclosamide powder into simple buffer}

As predicted in Figures S4 and S5 (Supplemental Information), and measured in Figs. $3 \mathrm{~A}$ and $3 \mathrm{~B}$, a $20 \mu \mathrm{M}$ niclosamide solution can readily be obtained by dissolving the "AK Sci polymorph" niclosamide powder into aqueous solution at a $\mathrm{pH}$ of 7.96. Similarly, a $200 \mu \mathrm{M}$ solution of niclosamide can be obtained in buffered solution at $\mathrm{pH} 9.01$; and the concentration can be raised to $300 \mu \mathrm{M}$ at $\mathrm{pH} 9.19$. The ultimate solubility of the negatively charged niclosamide salt $\left(\mathrm{Nic}_{-\mathrm{ve}}\right)$ at $\mathrm{pH} 9.63$ was at least $\sim 700 \mu \mathrm{M}$, i.e., total amount in solution $(703.6 \mu \mathrm{M})$ minus the intrinsic solubility of the acid $(2.53 \mu \mathrm{M})$.

For the nasal epithelium, while the average $\mathrm{pH}$ in the anterior (front) of the nose is 6.40 and the average $\mathrm{pH}$ in the posterior (back) of the nasal cavity is 6.27 , the overall range in $\mathrm{pH}$ is $\sim 5.17-8.13$ (43). Given that the $\mathrm{IC}_{100}$ concentration of niclosamide required to completely prevent viral replication in SARS-CoV-2 infected Calu-3 lung cells as measured by Ko et al. (35) as $\sim 2 \mu \mathrm{M}$, a $20 \mu \mathrm{M}$ niclosamide solution nasal spray is $10 \times$ this $\mathrm{IC}_{100}$. If testing in animals and humans reveals $20 \mu \mathrm{M}$ is safe, dose escalation is possible since, according to Fig. 3B, the niclosamide concentration can be increased to $30 \mu \mathrm{M}$ at $\mathrm{pH} 8.16$ and $50 \mu \mathrm{M}$ is obtained at only $\mathrm{pH} 8.39$. For the early treatment throat spray, concentrations can be escalated up to $300 \mu \mathrm{M}$ at an orally/ buccally tolerated $\mathrm{pH}$ of 9.19 , which is $\sim 150 \times$ the $\mathrm{IC}_{100}$. 
Thus, what has been learned is that, when niclosamide is present as a dissolved molecule in aqueous solution, its behavior is predictable by rudimentary concepts of solution chemistry. The theoretical maximum amount in solution is determined by the relative amounts of its pH-dependent unprotonated and protonated forms and follows the Henderson-Hasselbalch and precipitationpH (pHp) models (Figs. 3, and S4, S5, in Supplemental Information). Based on the fitted pKa of 7.12, which is in good agreement with the average of 6.52 from other reported values $(9,56-58)$, the amount of niclosamide in aqueous solution increases in the higher $\mathrm{pH}$ range (of 8-9.5). This is because the fractional amount of the more soluble deprotonated salt form is the dominant species. This pH-dependent behavior provides a mechanism for increasing the bioavailability of niclosamide in simple solutions for more direct application to the mucin-covered epithelia as nasal and throat sprays. Such formulations can be readily prepared simply by dissolving niclosamide powder into buffered aqueous solution, where dissolution times to relative equilibrium take $\sim 1 \mathrm{~h}$ in stirred vials (as in Fig. 5). Alternatively, and preferably for ultimate manufacturing, a concentrated ethanolic niclosamide can be made by solvent exchange into $\mathrm{pH}$ buffers to make the solutions in literally 1-2 s, as demonstrated in the rapid injection technique in Fig. 1. As pure solutions with no additional solid phase material, these solutions of niclosamide can be made up to at least $704 \mu \mathrm{M}$ at $\mathrm{pH} 9.63$.

\section{Some Supplied Polymorphs of Niclosamide Can Readily Revert to Their Most Stable Form, and So Reduce Their Solubility}

However, some supplied niclosamide already contains one or more polymorphic forms that can readily revert to their most stable form, and so reduce their absolute amounts in supernatant solution. Also, if, and when, precipitated or present as a particulate material, (e.g., micronized or surfactant- or protein- or polymer- stabilized microparticles as also observed in our provisional patent work (48)), the amount of niclosamide in aqueous solution is determined by the polymorphic nature of the material that the local aqueous solution is in equilibrium with, or is moving towards. This is where careful preformulation drug characterization is needed in order to fully understand and predict the nature of niclosamide's solid forms, its resulting intrinsic solubility in aqueous media, and hence its bioavailability, in (any) dosage form.

In support of these two major take a-ways, $(\mathrm{pH}$ dependent dissolution and reversion to more stable and lower solubility polymorphs) are the studies that explored the solid forms. That is:

- For one commercially available niclosamide powder (the "AK Sci polymorph"), after dissolution equilibrium was attained over a period of a few days at pH 9.3, the high concentration solution supernatant was, more often than not, stable despite being in contact with its original excess undissolved powder.

- For the "Sigma polymorph", as received, it already contained some of the monohydrate like material. While its, initially dissolved, high concentration solution was stable for a few hours, when left in contact with its original excess powder for longer periods, (e.g., overnight) it converted to a lower solubility solid morphology of needle-shaped crystals characteristic of the monohydrate reported by van Tonder et al. (2).

- For niclosamide precipitated from supersaturation the precipitated particles eventually achieved their own low solubility solid forms and showed a $\mathrm{pH}$ dependent morphology of flat particulate sheets to spiky needle-shaped crystals, again characteristic of the monohydrate (2).

- For the water-precipitate, and acetone and ethanol presumed "cosolvates", the solubilities were similarly low across the whole $\mathrm{pH}$ range.

A summary of this data is now shown in Fig. 15. Compared to the original AK Sci material (from Fig. 3B) are: the data from Fig. 6 (Niclosamide Precipitated from Supersaturated Solution); that from Fig. 12 (Sigma Niclosamide after $24 \mathrm{~h}$ ); and the final equilibrium amounts in solution measured for the water, acetone, and ethanol "cosolvates" (Fig. 9).

While still showing the expected $\mathrm{pH}$ dependence, i.e., dependent on the acid and salt solution equilibrium, there is a clear distinction and drop in amount of niclosamide in supernatant solution, dependent on the solid form in equilibrium with the species in solution. That is, for both the "Sigma polymorph" and the precipitated niclosamide materials, as well as the presumed "cosolvates", (i.e., the monohydrates and similar cosolvates identified by van Tonder et al. (2, $61)$ ), the amount of niclosamide in actual solution is dependent on the nature of the solid form it is in equilibrium with. (Unfortunately for the commercial powders, this information is not available because the manufacturers would not divulge their post synthesis processing to recover the niclosamide material as it is a trade secret). 
Fig. I5 Supernatant Niclosamide concentrations [Nic] $(\mu \mathrm{M})$ versus Supernatant $\mathrm{pH}$ for Dissolved-Equilibrated Niclosamide from AK Sci and Sigma, and Niclosamide precipitated from supersaturated solution plus the Water, Acetone, and Ethanol solvates at $\mathrm{pH}$ 9.3. The dotted line shows the $\mathrm{pHp}$ theory for $\mathrm{pKa}=7.12$ and the "AKSci polymorph" (Sol solubility = $2.53 \mu \mathrm{M}$ ) and the dashed line is for the presumed monohydrate So2 solubility $=1.01 \mu \mathrm{M}$.

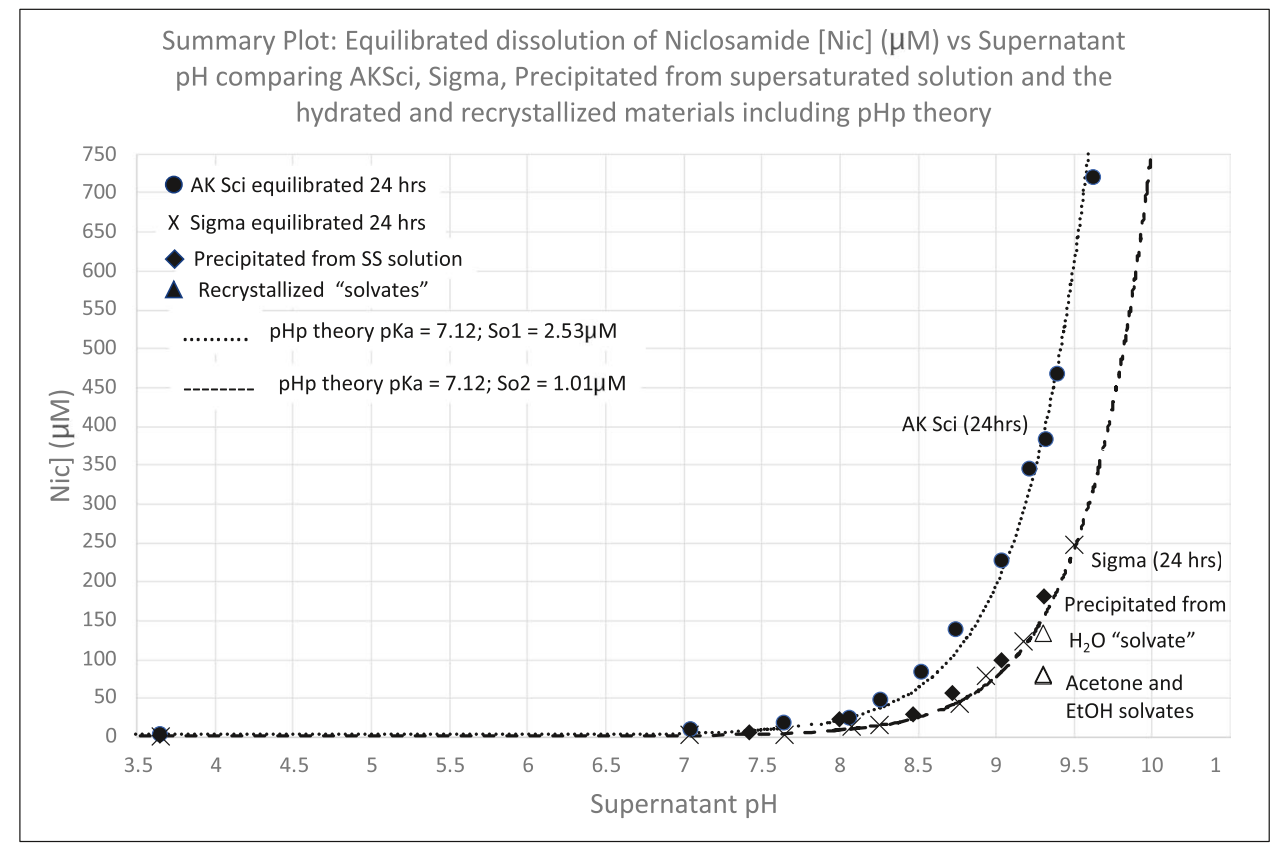

While beyond the scope of this current study, further studies by Raman and x-ray diffraction FT-IR/ Raman, and calorimetry could be carried out and are being planned to gain more insight into the crystal forms of these commercial products and the precipitates and solvates (62).

\section{SUMMARY AND CONCLUSIONS}

With the coronavirus pandemic still raging, more contagious (delta and now omicron) variants already in circulation (1), a world-wide average of only $40.6 \%$ fully vaccinated (52), and many parts of the world with only $4.2 \%$ vaccinated (73), there is still an urgent need for additional mitigation efforts to support current and potential vaccinations. Universal prophylactic nasal and early treatment throat sprays that "put the virus in lockdown", could help prevent infection and reduce viral load in the early stages for unvaccinated populations, as well as vaccinated spreaders where break through infections are now occurring. The niclosamide solutions presented here could represent such universal sprays.

Niclosamide has emerged from multiple drug screens with potential to treat cancer and many other diseases and conditions (22), and now in a broad range of viral infections (4-19). At the cellular level, niclosamide can inhibit three of the six stages of viral infection by: preventing uncoating and RNA release from the endosome (17); inhibiting viral replication by reducing the amount of ATP available from mitochondria needed for ATP-dependent viral transcription and translation (9); and interfering with capsid assembly in the Golgi that then promotes the secretion of non-competent virions (28). The main issue for nose and throat formulations, is how to optimize local bioavailability for a drug that has low intrinsic solubility in aqueous solution $(1-2 \mu \mathrm{M})$ compared to its cellular efficacy $(2-3 \mu \mathrm{M})$ and the fact that the traditional choice for nasal sprays of microparticles cannot penetrate the mucin. It is here that we have focused on what it takes to provide a mucin-penetrating niclosamide solution. The amount of niclosamide in aqueous buffered solution and therefore its bioavailability to infectable and infected epithelial cells, can be increased by just slightly increasing the solution pH (48).

Experimentation focused on a series of specific aims that measured the $\mathrm{pH}$-dependence of equilibrium supernatant concentrations and dissolution rates of niclosamide in buffered solutions. Niclosamide from different suppliers was compared, as well as precipitated niclosamide and presumed cosolvates of water, acetone and ethanol. UV/Vis spectroscopy was used to quantify niclosamide concentrations in supernatant solutions and data was compared to predictions from Henderson Hasselbalch and precipitation $\mathrm{pH}$ models. Optical microscopy was used to observe the morphologies of precipitated and converted niclosamide.

What has been learned is that, when niclosamide is present as a dissolved molecule in aqueous solution, its behavior is predictable by fundamental concepts in physical chemistry and pharmaceutics. Because it has a reported pKa of 5.6 to $7.2(9,56-58)$, and measured in this work to 
be 7.12, the theoretical maximum amount in solution is determined by the relative amounts of its $\mathrm{pH}$-dependent protonated and unprotonated forms. As shown here, the amount of niclosamide in supernatant solution follows the Henderson-Hasselbalch and precipitation-pH (pHp) models. Basically, for the high solubility "AK Sci polymorph" the amount of niclosamide in aqueous solution increases with increasing $\mathrm{pH}$, from $2.53 \mu \mathrm{M}$ at $\mathrm{pH} 3.66$, to $30 \mu \mathrm{M}$ at $\mathrm{pH}$ 8.16. It then showed a more rapid rise in concentration to $300 \mu \mathrm{M}$ at 9.16 , reaching $704 \mu \mathrm{M}$ at $\mathrm{pH} 9.63$. This rise in the amount in solution at these slightly alkaline $\mathrm{pHs}$ was because the fractional amount of the more soluble deprotonated salt form is the dominant species. This $\mathrm{pH}$ dependent behavior therefore provides a mechanism for increasing the bioavailability of niclosamide in simple solutions for more direct application as nasal and throat sprays. Such formulations can be readily prepared by dissolving niclosamide powder into buffered aqueous solution, or preferably mixed by solvent exchange from concentrated ethanolic niclosamide into $\mathrm{pH}$ buffers.

However, the cautionary tale is that if and when precipitated or present as a particulate material, micronized or surfactant- or protein- or polymer- "stabilized" microparticles, the amount of niclosamide in aqueous solution is determined by the nature of the material that the local aqueous solution is in equilibrium with, or is moving towards. Thus, in the preparation of any formulation of niclosamide, especially for local nasal and throat delivery, niclosamide is not niclosamide is not niclosamide, i.e., the source and nature of niclosamide can dramatically influence the polymorphism, solubility, amount in solution, bioavailability, and pharmaceutical performance of the drug compound.

As now reformulated, and when tested as a nasal and throat spray with optimized bioavailability, niclosamide could potentially represent a universal prophylactic and early treatment for many viral infections, including ones that mutate and escape current vaccine protection. Formulated as an optimized mucin-penetrating solution, niclosamide can potentially help to control initial infection and viral loads across the board, while vaccines and other oral antivirals provide subsequent immune protection and additional reductions in viral load. As with vaccine development, there is a need for billions of doses, (especially on a frequent basis) and so there is certainly room for several prophylactic formulations, tested and produced by multiple governments, infectious disease institutes, schools of public health, and companies that can help to control such initial viral infection and early symptoms. Our niclosamide solution formulation has already been show that it can be scaled up to multi-liter volumes as a 503b pharmacy batch that can be provided in $10 \mathrm{~mL}$ sealed and capped sterile vials. "Why Niclosamide?", and its intracellular virostatic effects, along with how these measurements are translated into spray products and preclinical and clinical outcomes are discussed in more detail in a white paper "Perspective" currently in preparation (25).

Our lead niclosamide formulation is therefore a simple buffered solution that represents a potentially universal prophylactic nasal spray that could stop virus replication at its point of entry, and a higher concentration throat spray, that could reduce viral load as it progresses down the back of the throat. A low dose $(20 \mu \mathrm{M})$ prophylactic solution of niclosamide at a nasally safe and acceptable $\mathrm{pH}$ of 7.96 and a throat spray of up to $300 \mu \mathrm{M}$ at $\mathrm{pH} 9.19$ would be the simplest and potentially most effective formulations from both an efficacy standpoint as well as manufacturing and distribution, with no cold chain. They now just need testing.

\section{FUNDING STATEMENT}

There was no formal funding from government (e.g., NIH) or other agency. Supplies were paid for from a discretionary account. Regarding salaries, the sole person engaged in all of the work was Professor David Needham PhD, DSc, employed at Duke University in the Pratt School of Engineering in the Department of Mechanical Engineering and Material Science.

Supplementary Information The online version contains supplementary material available at https://doi.org/10. 1007/s11095-021-03112-x.

\section{Acknowledgements and Disclosures}

Acknowledged are the important discussions with Professors Phil Williams and Jonathan Burley at the School of Pharmacy at the University of Nottingham, UK, and Dr. Carla Gene Rapp, who helped to shape the thinking, interpretation of results, and overall flow of the manuscript. Also, thanks are to Peter Silinski of the Duke Analytical Instrument Facility who analysed niclosamide purity and stability.

\section{Declarations}

Conflict of Interest Statement Duke University has assigned the rights for the provisional patent applications to David Needham and his entity, Needham Material Science LLC.

Author contributions for all authors David Needham was the sole contributor and sole author.

Data Availability Statement The datasets generated during and/ or analyzed during the current study are available from the corresponding author on reasonable request. 


\section{REFERENCES}

1. CDC. SARS-CoV-2 Variant Classifications and Definitions https://www.cdc.gov/coronavirus/2019-ncov/variants/varia nt-info.html 2021. Accessed on 2021-11-21.

2. van Tonder EC, Maleka TSP, Liebenberg W, Song M, Wurster DE, de Villiers MM. Preparation and physicochemical properties of niclosamide anhydrate and two monohydrates. Int J Pharm. 2004;269(2):417-32.

3. Brunaugh AD, Seo H, Warnken Z, Ding L, Seo SH, Smyth HDC. Development and evaluation of inhalable composite niclosamide-lysozyme particles: A broad-spectrum, patientadaptable treatment for coronavirus infections and sequalae. PloS One. 2021;16(2):e0246803-e.

4. Braga L, Ali H, Secco I, Chiavacci E, Neves G, Goldhill D, et $a l$. Drugs that inhibit TMEM16 proteins block SARS-CoV-2 spike-induced syncytia. Nature. 2021;594(7861):88-93.

5. Cabrita I, Benedetto R, Schreiber R, Kunzelmann K. Niclosamide repurposed for the treatment of inflammatory airway disease. JCI Insight. 2019;4(15).

6. Cheng B, Morales LD, Zhang Y, Mito S, Tsin A. Niclosamide induces protein ubiquitination and inhibits multiple prosurvival signaling pathways in the human glioblastoma U-87 MG cell line. PloS One. 2017;12(9):e0184324-e.

7. Imperi F, Massai F, Ramachandran Pillai C, Longo F, Zennaro E, Rampioni G, et al. New life for an old drug: the anthelmintic drug niclosamide inhibits Pseudomonas aeruginosa quorum sensing. Antimicrob Agents Chemother. 2013;57(2):996.

8. Jeon S, Ko M, Lee J, Choi I, Byun SY, Park S et al. Identification of antiviral drug candidates against SARS-CoV-2 from FDA-approved drugs. bioRxiv. 2020:2020.03.20.999730.

9. Jurgeit A, McDowell R, Moese S, Meldrum E, Schwendener R, Greber UF. Niclosamide Is a Proton Carrier and Targets Acidic Endosomes with Broad Antiviral Effects. PLOS Pathogens. 2012;8(10):e1002976.

10. Kadri H, Lambourne OA, Mehellou Y. Niclosamide, a Drug with Many (Re)purposes. ChemMedChem. 2018;13(11):1088-91.

11 Khanim FL, Merrick BAME, Giles HV, Jankute M, Jackson JB, Giles LJ, et al. Redeployment-based drug screening identifies the anti-helminthic niclosamide as anti-myeloma therapy that also reduces free light chain production. Blood Cancer J. 2011;1:e39.

12. Liu C, Lou W, Zhu Y, Nadiminty N, Schwartz CT, Evans $\mathrm{CP}$, et al. Niclosamide inhibits androgen receptor variants expression and overcomes enzalutamide resistance in castration-resistant prostate cancer. Clin Cancer Res. 2014;20(12):3198-210.

13. McCollum CW, Conde-Vancells J, Hans C, Vazquez-Chantada M, Kleinstreuer N, Tal T, et al. Identification of vascular disruptor compounds by analysis in zebrafish embryos and mouse embryonic endothelial cells. Reprod Toxicol. 2017;70:60-9.

14. Miner K, Labitzke K, Liu B, Wang P, Henckels K, Gaida K, et al. Drug Repurposing: The Anthelmintics Niclosamide and Nitazoxanide Are Potent TMEM16A Antagonists That Fully Bronchodilate Airways. Front Pharmacol. 2019;10(51).

15. Mook RA, Zhao S, Chen W. Abstract 5491: Interrogating the mechanism of Wnt pathway inhibition by niclosamide. Can Res. 2014;74(19 Supplement):5491.

16. Mostafa A, Kandeil A, Elshaier AMMY, Kutkat O, Moatasim Y, Rashad AA, et al. FDA-Approved Drugs with Potent In Vitro Antiviral Activity against Severe Acute Respiratory Syndrome Coronavirus 2. Pharmaceuticals. 2020;13(12).
17. Prabhakara C, Godbole R, Sil P, Jahnavi S, van Zanten TS, Sheth D, et al. Niclosamide inhibits SARS-CoV2 entry by blocking internalization through pH-dependent CLIC/ GEEC endocytic pathway. bioRxiv. 2020:2020.12.16.422529.

18. Schweizer MT, Haugk K, McKiernan JS, Gulati R, Cheng HH, Maes JL, et al. A phase I study of niclosamide in combination with enzalutamide in men with castration-resistant prostate cancer. PLOS ONE. 2018;13(6):e0198389.

19. Xu J, Shi P-Y, Li H, Zhou J. Broad Spectrum Antiviral Agent Niclosamide and Its Therapeutic Potential. ACS Infect Dis. 2020;6(5):909-15.

20. Bayer. YOMESAN® tablets (MSDS) https://www.bayer.co. $\mathrm{za} /$ static/documents/MSDS/PIs/YOMESAN_EN_PI.pdf. 2008 Accessed on 2021-11-21.

21. Abrams GJ, Seftel HC, Heinz HJ. The treatment of human tapeworm infections with 'Yomesan.' S Afr Med J. 1963;37:6-8.

22. Chen W, Mook RA, Premont RT, Wang J. Niclosamide: Beyond an antihelminthic drug. Cell Signal. 2018;41:89-96.

23. Goulding J. Virus Replication https://www.immunology. org/public-information/bitesized-immunology/pathogensand-disease/virus-replication. British Society for Immunology 2021. Accessd on 2021-11-21.

24. Laise P, Bosker G, Sun X, Shen Y, Douglass EF, Karan C, et $a l$. The Host Cell ViroCheckpoint: Identification and Pharmacologic Targeting of Novel Mechanistic Determinants of Coronavirus-Mediated Hijacked Cell States. bioRxiv : the preprint server for biology. 2020:2020.05.12.091256.

25. Needham D. A White Paper. Why Niclosamide? A Basis for New Nasal and Throat Spray Solution-Formulation for COVID19 and Other Viral Infections. In preparation, to be submitted to Pharmaceutical Research. 2021.

26. Huang Y, Chen S, Yang Z, Guan W, Liu D, Lin Z, et al. SARSCoV-2 Viral Load in Clinical Samples from Critically Ill Patients. Am J Respir Crit Care Med. 2020;201(11):1435-8.

27. Shang J, Wan Y, Luo C, Ye G, Geng Q, Auerbach A, et al. Cell entry mechanisms of SARS-CoV-2. Proc Natl Acad Sci U S A. 2020;117(21):11727-34.

28. Jung E, Nam S, Oh H, Jun S, Ro H-J, Kim B, et al. Neutralization of Acidic Intracellular Vesicles by Niclosamide Inhibits Multiple Steps of the Dengue Virus Life Cycle In Vitro. Sci Rep. 2019;9(1):8682.

29. Conaway RC, Conaway JW. ATP activates transcription initiation from promoters by RNA polymerase II in a reversible step prior to RNA synthesis. J Biol Chem. 1988;263(6):2962-8.

30. Zimmerman JJ, von Saint André-von Arnim A, McLaughlin J. Chapter 74 - Cellular Respiration. In: Fuhrman BP, Zimmerman JJ, editors. Pediatric Critical Care (Fourth Edition). Saint Louis: Mosby; 2011. p. 1058-72.

31. Promega. CellTiter-Glo® 2.0 Cell Viability Assay. https:// www.promega.com/products/cell-health-assays/cell-viabi lity-and-cytotoxicity-assays / celltiter_glo-2_0-assay. 2021 (Accessd on 2021-11-21).

32. Chang C-W, Li H-C, Hsu C-F, Chang C-Y, Lo S-Y. Increased ATP generation in the host cell is required for efficient vaccinia virus production. J Biomed Sci. 2009;16(1):80-.

33. Nagy PD, Lin W. Taking over Cellular Energy-Metabolism for TBSV Replication: The High ATP Requirement of an RNA Virus within the Viral Replication Organelle. Viruses. 2020;12:56.

34. Kim S, Bear S, Needham D, Lee P. Effect of Niclosamide on cytotoxicity and cell viability in small airway epithelial cells in preparation. 2021.

35. Ko M, Jeon S, Ryu W-S, Kim S. Comparative analysis of antiviral efficacy of FDA-approved drugs against SARS-CoV-2 in 
human lung cells: Nafamostat is the most potent antiviral drug candidate. bioRxiv. 2020:2020.05.12.090035.

36. clinicaltrials.gov. COVID19 Niclosamide https://clinicaltr ials.gov/ct2/ results? term=Niclosamide\&cond=Covid 19 . 2021 Accessd on 2021-11-21.

37. Daewoong-Pharmaceutical. Daewoong Pharmaceutical Partners with Tufts Medical Center for Phase 2 Clinical Trial with Niclosamide. https://www.prnewswire.com/ news-releases/daewoong-pharmaceutical-partners-withtufts-medical-center-for-phase-2-clinical-trial-with-niclo samide-301162739.html. 2020 Accessed on 2021-11-21.

38. Union-Therapeutics. UNION's nasal spray for COVID-19 enters a 1,500 high-risk patient study. 2021.

39. Boegh M, Nielsen HM. Mucus as a barrier to drug delivery - understanding and mimicking the barrier properties. Basic Clin Pharmacol Toxicol. 2015;116(3):179-86.

40. Leal J, Smyth HDC, Ghosh D. Physicochemical properties of mucus and their impact on transmucosal drug delivery. Int J Pharm. 2017;532(1):555-72.

41. Paul AD, Samatha P, Manasa SL, Munemma R, Supriya D. Modeling the oral cavity with mucoadhesive drug delivery systems - a potential alternative to conventional therapy. Int J Pharm Sci Drug Res. 2017;9:299-307.

42. Hou YJ, Okuda K, Edwards CE, Martinez DR, Asakura T, Dinnon KH 3rd, et al. SARS-CoV-2 Reverse Genetics Reveals a Variable Infection Gradient in the Respiratory Tract. Cell. 2020;182(2):429-46.e14.

43. Washington N, Steele RJ, Jackson SJ, Bush D, Mason J, Gill $\mathrm{DA}$, et al. Determination of baseline human nasal $\mathrm{pH}$ and the effect of intranasally administered buffers. Int J Pharm. 2000;198(2):139-46.

44. Shah BAK. FLONASE (fluticasone propionate) nasal spray. Prescribing information. In: DPARP, editor. 2019.

45. Baldwin CM, Scott LJ. Mometasone furoate: a review of its intranasal use in allergic rhinitis. Drugs. 2008;68(12):1723-39.

46. Merck. Prescribing information Nasonex. 1997.

47. Jones N. The nose and paranasal sinuses physiology and anatomy. Adv Drug Deliv Rev. 2001;51(1):5-19.

48. Needham D, inventor; Duke University, assignee. Current Provisional Patent Portfolio USPO: a) DU7305PROV, US 63/111,184 "ANTI-VIRAL NICLOSAMIDE COMPOSITIONS AS VIROSTATIC THERAPEUTICS AND METHODS OF MAKING AND USING SAME” (filed 11/9/2020). b) DU7185PROV, US 63/049,160, "NICLOSASPRAY" AND "NICLOSADROPS" FOR BUCCAL/THROAT, NASAL, AND EYE DROPS AND METHODS OF USING SAME" (filed 7/8/2020). c) DU7175PROV, US 63/049,153, "ANTIVIRAL NICLOSAMIDE COMPOSITIONS AS VIROSTATIC THERAPEUTICS AND METHODS OF MAKING AND USING SAME” (filed 7/8/2020). d) DU7109PROV, US 63/021,250, "COMPOSITIONS COMPRISING NICLOSAMIDE AND METHODS OF USING SAME AS PROPHYLAXIS AND TREATMENT OF VIRAL INFECTIONS" (filed $5 / 7 / 2020)$. e) DU6187PROV, US 63/021,241, PRECIPITATION OF HYDROPHOBIC DRUGS AS NANOCRYSTALS FOR INJECTION TO A SUBJECT AND METHODS OF USING SAME (filed 5/72020). f) DU7099PROV, US 63/014,748, "COMPOSITIONS COMPRISING NICLOSAMIDE AND METHODS OF MAKING AND USING SAME FOR THE PROPHYLAXIS AND TREATMENT OF VIRAL INFECTIONS" (filed 4/24/2020). g) DU7065PROV2, US 63/111,785, "COMPOSITIONS AND FORMULATIONS COMPRISING NICLOSAMIDE AND METHODS OF MAKING AND USING SAME FOR THE TREATMENT OF VIRAL INFECTIONS" (filed 4/17/2020). h) DU7065PROV,
US 63/006,527, "NICLOSAMIDE COMPOSITIONS AND METHODS OF USING SAME FOR THE TREATMENT OF VIRAL INFECTIONS" (filed 4/7/2020). i) DU7308PROV, US 63/007,007, "ANTI-VIRAL NICLOSAMIDE COMPOSITIONS AS VIROSTATIC THERAPEUTICS AND METHODS OF MAKING AND USING SAME” (filed 3/26/2020). USA2020.

49. Jeon S, Ko M, Lee J, Choi I, Byun SY, Park S, et al. Identification of Antiviral Drug Candidates against SARS-CoV-2 from FDA-Approved Drugs. Antimicrob Agents Chemother. 2020;64(7):e00819-e820.

50. CDC. COVID-19 Vaccine Breakthrough Infections Reported to CDC - United States, January 1-April 30, 2021, https://www.cdc.gov/mmwr/volumes/70/wr/ mm7021e3.htm. 2021 Accessed on 2021-11-21.

51. Delta Variant Key to Breakthrough Infections in Vaccinated Israelis [press release]. Medscape2021.

52. Ourworldindata. Share of people vaccinated against COVID-19, Jul 23, 2021. 2021.

53. Hernandez J. Nearly 94,000 Kids Got COVID-19 Last Week. They Were 15\% Of All New Cases (August 10 2021). NPR; 2021.

54. Needham D, Arslanagic A, Glud K, Hervella P, Karimi L, Høeilund-Carlsen P-F, et al. Bottom up design of nanoparticles for anti-cancer diapeutics. J Drug Target. 2016;24(9):836-56.

55 Reddy GB, Kerr DL, Spasojevic I, Tovmasyan A, Hsu D, Brigman BE, et al. Preclinical Testing of a Novel Niclosamide Stearate Prodrug Therapeutic (NSPT) shows efficacy against Osteosarcoma. Mol Cancer Ther. 2020;19(7):1448.

56. FAO. Food and Agriculture Organization of the United Nations (FAO) SPECIFICATIONS AND EVALUATIONS FOR AGRICULTURAL PESTICIDES NICLOSAMIDE 2',5-dichloro-4'-nitrosalicylanilide 2004.

57. McConville MB, Hubert TD, Remucal CK. Direct Photolysis Rates and Transformation Pathways of the Lampricides TFM and Niclosamide in Simulated Sunlight. Environ Sci Technol. 2016;50(18):9998-10006.

58. Tran TH, Labanowski J, Gallard H. Adsorption and transformation of the anthelmintic drug niclosamide by manganese oxide. Chemosphere. 2018;201:425-31.

59. Caira MR, Van Tonder EC, De Villiers MM, Lötter AP. Diverse Modes of Solvent Inclusion in Crystalline Pseudopolymorphs of the Anthelmintic Drug Niclosamide. J Inclusion Phenom Mol Recognit Chem. 1998;31(1):1-16.

60. de Villiers MM, Mahlatji MD, van Tonder EC, Malan SF, Lötter AP, Liebenberg W. Comparison of the physical and chemical stability of niclosamide crystal forms in aqueous versus nonaqueous suspensions. Drug Dev Ind Pharm. 2004;30(6):581-92.

61 van Tonder EC, Mahlatji MD, Malan SF, Liebenberg W, Caira MR, Song M, et al. Preparation and physicochemical characterization of 5 niclosamide solvates and 1 hemisolvate. AAPS PharmSciTech. 2004;5(1):E12-E.

62. Burley J, Needham D. Characterization of Niclosamide (XRD, FT-IR/Raman, DSC) from various suppliers and as precipitates and cosolvates confirms polymorphic forms as a function of $\mathrm{pH}$ (in preparation). 2021.

63 Halász G, Gyüre B, Jánosi IM. Vortex flow generated by a magnetic stirrer. Am J Phys. 2007;75:1092.

64. Duncan PB, Needham D. Test of the Epstein-Plesset model for gas microparticle dissolution in aqueous media: Effect of surface tension and gas undersaturation in solution. Langmuir. 2004;20(7):2567-78.

65. Duncan PB, Needham D. Microdroplet dissolution into a second-phase solvent using a micropipet technique: Test of 
the Epstein-Plesset model for an aniline-water system. Langmuir. 2006;22(9):4190-7.

66. Epstein PS, Plesset MS. On the Stability of Gas Bubbles in Liquid-Gas Solutions. J Chem Phys. 1950;18(11):1505-2409.

67. Su JT, Needham D. Mass transfer in the dissolution of a multicomponent liquid droplet in an immiscible liquid environment. Langmuir. 2013;29(44):13339-45.

68. Kim DH, Costello MJ, Duncan PB, Needham D. Mechanical properties and microstructure of polycrystalline phospholipid monolayer shells: Novel solid microparticles. Langmuir. 2003;19(20):8455-66.

69. Kim DH, Klibanov AL, Evans EA, Needham D. Viscoelastic properties of phospholipid monolayer shells on air microbubbles. Biophys J. 1998;74(2):A313-A.

70. Llinàs A, Box KJ, Burley JC, Glen RC, Goodman JM. A new method for the reproducible generation of polymorphs: two forms of sulindac with very different solubilities. J Appl Crystallogr. 2007;40(2):379-81.
71. Jara MO, Warnken ZN, Sahakijpijarn S, Moon C, Maier EY, Christensen DJ, et al. Niclosamide Inhalation Powder Made by Thin-Film Freezing: Pharmacokinetic and Toxicology Studies in Rats and Hamsters. bioRxiv. 2021:2021.01.26.428293.

72. Union-Therapeutics. UNION therapeutics A/S completes dosing of healthy volunteers with UNI911 (inhaled niclosamide) for COVID-19 https://www.uniontherapeutics.com/ news-events / news/union-therapeutics-a-s-completes-dosingof-healthy-volunteers-with-uni911-inhaled-niclosamide-forcovid-19. 2020 Accessed on 2021-11-21.

73. CDC. Global COVID-19 Vaccinations https://covid.cdc.gov/ covid-data-tracker/\#global-vaccinations. 2021 Accessed on 2021-11-21.

Publisher's Note Springer Nature remains neutral with regard to jurisdictional claims in published maps and institutional affiliations. 